Copyright (C) 2006 IEEE. Reprinted from IEEE Transactions on Wireless Communications, 2004; 3 (1):300-314

This material is posted here with permission of the IEEE. Such permission of the IEEE does not in any way imply IEEE endorsement of any of the University of Adelaide's products or services. Internal or personal use of this material is permitted. However, permission to reprint/republish this material for advertising or promotional purposes or for creating new collective works for resale or redistribution must be obtained from the IEEE by writing to pubs-permissions@ieee.org.

By choosing to view this document, you agree to all provisions of the copyright laws protecting it. 


\title{
Receivers With Chip-Level Decision Feedback Equalizer for CDMA Downlink Channels
}

\author{
Jinho Choi, Senior Member, IEEE, Seong Rag Kim, Member, IEEE, and Cheng-Chew Lim, Senior Member, IEEE
}

\begin{abstract}
For the code division multiple access (CDMA) downlink channel, we investigate the decision feedback equalizer (DFE) at chip-level to effectively suppress multiple-access interference (MAI) when the spreading sequences are orthogonal. The structure of the receiver with the chip-level DFE has been investigated and the minimum mean-square error solution has been derived. Due to the inherent structure of the chip-level DFE, some iterative techniques with hard and soft decisions have been proposed. It is shown that the proposed receivers with the chip-level DFE can provide satisfactory performance. In comparison with the adaptive chip-level linear equalizer, the number of users can be doubled by using adaptive chip-level DFE at a bit-error rate of $10^{-3}$. Throughout the paper, we assume that scrambled orthogonal codes are used for spreading sequences.
\end{abstract}

Index Terms-Code-division multiple-access (CDMA), decision feedback equalizer (DFE), downlink, interference suppression, iterative technique.

\section{INTRODUCTION}

$\mathbf{S}$ INCE THE performance of code-division multiple-access (CDMA) systems is limited by the multiple-access interference (MAI), joint detection, or multiuser detection has been extensively investigated to mitigate the MAI [18]. Generally, the multiuser receiver can perform much better than the conventional correlator based receiver, at the expense of increased complexity. For uplink, the increase of complexity may not be a big issue, because the base station can be equipped with powerful computing processors. On the other hand, since the mobile terminal is certainly limited by cost and size, it would be difficult to use the multiuser receiver for the downlink. However, regardless of this difficulty, there is strong demand to improve the performance of the downlink for asymmetric high-speed wireless data services (e.g., wireless Internet).

The multiuser receiver with a linear filter becomes possible when the spreading codes are periodic (i.e., short codes) with adaptive techniques [15] (see [8] for an overview). When the spreading codes are periodic, the received signal becomes cyclostationary and the optimal filter vector does not differ from

Manuscript received April 19, 2002; revised September 12, 2002 and December 12, 2002; accepted January 8, 2003. The editor coordinating the review of this paper and approving it for publication is G. Leus.

J. Choi is with the School of Electrical Engineering and Telecommunications, The University of New South Wales (UNSW), Sydney NSW 2052, Australia (e-mail: j.choi@unsw.edu.au).

S. R. Kim is with the Mobile Telecommunications Research Laboratory, Electronic and Telecommunications Research Institute (ETRI), Taejon 305-600, Korea (e-mail: srkim@etri.re.kr).

C.-C. Lim is with the School of Electrical and Electronic Engineering, University of Adelaide, Adelaide 5005, Austrialia (e-mail: cheng.lim@ adelaide.edu.au).

Digital Object Identifier 10.1109/TWC.2003.819029 symbol to symbol. From this, adaptive algorithms can be readily applied to find (or estimate) the optimal solution adaptively with low computational burden. In [9], a blind adaptive receiver has been investigated with the assumption of knowing the spreading code of the desired user only. For a frequency-selective fading channel, the blind adaptive receiver is further generalized in [19] and [20]. To track the variation of the channel in a timevariant fading environment, the rake structure has been employed [2],[12]. However, since most CDMA standards use aperiodic spreading codes, it is difficult to use the above adaptive techniques, because the optimal weight vector shall be different from symbol to symbol due to different spreading codes for each symbol.

CDMA downlink has two interesting features: One is that all transmissions are synchronized and the other is that the (short or long) spreading codes can be orthogonal. By taking advantage of these features, the chip-level equalization was proposed to mitigate the MAI together with a despreader [4], [10]. A despreader can mitigate the MAI after chip-level equalization to restore the orthogonality. As shown in [14], this receiver (with a chip-level equalizer) can be easily implemented with adaptive algorithms.

Unfortunately, the performance of the receiver with a chip-level linear equalizer (LE) is not significantly better than the rake receiver, unless the receiver of the mobile station is equipped with multiple receiving antennas or uses oversampling. Since the LE cannot perfectly remove the multipath interference (MPI) with a single receiving antenna and chip-rate sampling, there exists residual MPI. Hence, the MAI cannot be completely suppressed by the correlator (or despreader). To avoid this, multiple receiving antennas or a higher sampling rate can be used [11], [14] for the chip-level minimum mean-square error (MMSE) LE. Note that the chip-level MMSE LE in [14] is different from that in [11], although the LE in both cases operates at the chip-level. In [11], a chip-level MSE cost function has been used, while a symbol-level MSE cost function was used in [14]. Although the chip-level MSE cost function is conceptually convenient to derive a closed-form solution of the chip-level MMSE LE, it would not be directly used for adaptive implementation due to the updating at the chip rate. On the other hand, since the symbol-level cost function in [14] allows the updating at the symbol rate with code-multiplexed pilot, it would be practical.

In this paper, we investigate using the chip-level decision feedback equalizer (DFE) to achieve better performance than the chip-level LE. We assume that scrambled orthogonal spreading codes are used. The symbol-level MSE cost function is used, as in [14]. For short spreading codes, the DFE was considered previously [1], [16], where the DFE consisted of 
a feedforward filter (FFF) at the chip rate (or higher) and a feedback filter (FBF) at the symbol rate. The role of the FFF is the same as that of the linear filter in [15], and is sampled at the symbol rate for the FBF, which is used to remove postcursor intersymbol interference (ISI) at the symbol level. The filter coefficients of the FFF and FBF are determined to minimize symbol-level MSE. It is possible to use the DFE for long spreading codes. In this case, however, the FBF should be used at the chip rate. If the FBF is used at symbol rate, the optimal filter coefficients of the FBF vary from symbol to symbol. This complicates the use of adaptive algorithms. Hence, we use the FBF at the chip rate in this paper so that adaptive algorithms can be used to find the filter coefficients of the DFE.

We derive a closed-form solution of the optimal chip-level MMSE DFE. Due to the inherent structure of the chip-level DFE, iterative receivers have been proposed using some adaptive approaches with a code-multiplexed pilot to determine the filter coefficient adaptively. Iterative techniques can improve the performance of the chip-level DFE significantly. For better adaptation, the use of soft decision is also investigated. These are the main contributions of the paper.

The rest of the paper is organized as follows. In Section II, we consider the system model for a CDMA downlink. Chip-level equalizers and the rake receiver are derived for a CDMA downlink in Section III. A closed-form expression of the optimal solution of the chip-level MMSE DFE is shown (in Appendix A). Due to the inherent structure of the chip-level DFE, we devise some iterative methods for the feedback in Section IV. Hard and soft decisions are available for the feedback. It is shown that the soft decision has an advantage in effective updating of the chip-level DFE to track time-variant channels. Computer simulation results are presented in Section $\mathrm{V}$ to see the performance and we conclude the paper with some remarks in Section VI.

\section{SySTEM MODEL}

Let $N$ be the processing gain. Denote by $c_{(k), N l+m}$ the normalized spreading sequence for user $k$. The indexes $l$ and $m$ are used for the symbol index and the chip index within a symbol period, respectively. We assume that the spreading sequences are orthogonal to each other, i.e.,

$$
\sum_{m=0}^{N-1} c_{(k), N l+m} c_{\left(k^{\prime}\right), N l+m}^{*}=\delta_{k, k^{\prime}}
$$

where $\delta_{k, k^{\prime}}$ is the Kronecker delta. These spreading sequences can be simply generated by scrambling Walsh codes with a pseudo-random complex-binary ${ }^{1}$ sequence. In this case, the receiver can easily know all other users' spreading codes. The spread signal for the CDMA downlink channel is written as

$$
x_{N l+m}=\sum_{k=1}^{K} c_{(k), N l+m} b_{(k)}(l), \quad m=0,1, \ldots, N-1
$$

where $b_{(k)}(l)$ stands for the $l$ th symbol of transmission $k$ (to mobile terminal $k$ ) and $K$ is the number of transmissions. Throughout the paper, we assume that the first transmission is

\footnotetext{
${ }^{1} \mathrm{~A}$ random complex-binary sequence consists of $\{( \pm 1 \pm i) / \sqrt{2}\}$, where $i=\sqrt{-1}$.
}

the pilot signal (i.e., we use a code-multiplexed pilot). Suppose that there are $L$ receiving antennas for the mobile station. The received signal from a frequency-selective fading channel is written as

$$
y_{j, N l+m}=\sum_{p=0}^{L_{h}} h_{j, p ; l} x_{N l+m-p}+n_{j, N l+m}, \quad j=1,2, \ldots, L
$$

where $h_{j, p ; l}$ denotes the channel impulse response (CIR) from the transmit antenna to receiving antenna $j, L_{h}+1$ is the length of the CIR, and $n_{j, N l+m}$ is the background complex white Gaussian noise of receiving antenna $j$ with zero mean and variance of $N_{0}$. Note that due to the cost and size of the mobile station, it would be hard to increase $L$ in general.

\section{RECEIVERS FOR CDMA DOWNLINK}

In this section, we consider the rake receiver and the receiver with a chip-level equalizer for the CDMA downlink channel. We assume that the receiver knows the spreading sequences of all active users.

\section{A. Rake Receiver}

Utilizing code-multiplexed pilot, the instantaneous channel estimate of $h_{j, p ; l}$ is given by

$$
\begin{aligned}
\hat{h}_{j, p ; l} & =\sum_{m=0}^{N-1} y_{j, N l+m+p} c_{(1), N l+m}^{*} b_{(1)}^{*}(l) \\
p & =0,1, \ldots, L_{h}, \quad j=1,2, \ldots, L .
\end{aligned}
$$

The instantaneous channel estimate can be smoothed with zero lag for a better estimate to give

$$
\overline{\hat{h}}_{j, p ; l}=\frac{1}{B} \sum_{q=0}^{B-1} \hat{h}_{j, p ; l-q}, \quad p=0,1, \ldots, L_{h}
$$

where $B$ is the size of the moving window. With these channel estimates, the output of the maximal ratio combining (MRC) rake receiver for transmission $k$ can be written as

$$
\begin{aligned}
\hat{b}_{\text {rake, }(k)}(l) & =\sum_{j=1}^{L} \sum_{p=0}^{L_{h}} \overline{\hat{h}}_{j, p ; l}^{*}\left(\sum_{m=0}^{N-1} y_{j, N l+m+p} c_{(k), N l+m}^{*}\right) \\
k & =2,3, \ldots, K .
\end{aligned}
$$

\section{B. Receiver With Chip-Level MMSE DFE}

In this section, we assume that the channel coefficients are time-invariant (i.e., $h_{j, p ; l}=h_{j, p}$ for all $l$ ) for the chip-level DFE shown in Fig. 1.

Suppose that the decided symbols are available for the FBF in the chip-level DFE as $\tilde{b}_{(k)}(l), k=2,3, \ldots, K$. Note that $b_{(1)}(l)$ is known, because the first transmission is the code-multiplexed pilot. Then, the input to the FBF to remove the postcursor MPI is written as

$$
\begin{gathered}
\tilde{x}_{N l+m}=\sum_{k=1}^{K} c_{(k), N l+m} \tilde{b}_{(k)}(l) \\
m=0,1, \ldots, N-1
\end{gathered}
$$

where $\tilde{b}_{(1)}(l)=b_{(1)}(l)$. Let $\left\{g_{j, p}\right\}$ and $\left\{f_{p}\right\}$ be the impulse responses of the $j$ th FFF and the FBF of the chip-level DFE, 


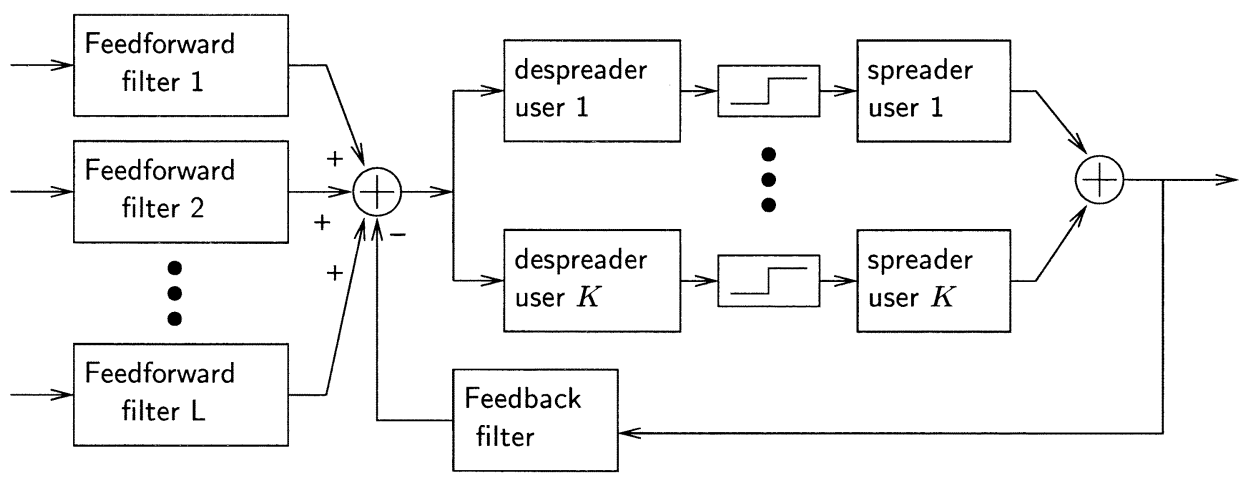

Fig. 1. Block diagram of the receiver with chip-level DFE.

respectively. Let $\left(L_{g}+1\right)$ be the length of each FFF. Then, the output of the chip-level DFE is written as

$$
\begin{aligned}
\hat{x}_{N l+m}= & \sum_{j=1}^{L} \sum_{p=-L_{g}}^{0} g_{j, p} y_{j, N l+m-p}-\sum_{p=1}^{L_{h}} f_{p} \tilde{x}_{N l+m-p} \\
= & \sum_{j=1}^{L} \sum_{p=-L_{g}}^{L_{h}} v_{j, p} x_{N l+m-p}-\sum_{p=1}^{L_{h}} f_{p} \tilde{x}_{N l+m-p} \\
& +w_{N l+m}, \quad m=0,1, \ldots, N-1
\end{aligned}
$$

where $\left\{v_{j, p}\right\}_{p=-L_{q}}^{L_{h}}$ is the impulse response of the composite system of the $j$ th channel (from the transmitting antenna to receiving antenna $j$ ) and the $j$ th FFF and $w_{N l+m}$ is the sum of the noise terms obtained from $n_{j, N l+m}$ after passing through the $j$ th FFF. If the equalization is perfect, the estimate of the symbol for the $k$ th transmission can be found to be

$$
\hat{b}_{(k)}(l)=\sum_{m=0}^{N-1} \hat{x}_{N l+m} c_{(k), N l+m}^{*} .
$$

In general, $\tilde{b}_{(k)}(l)=\operatorname{dec}\left(\hat{b}_{(k)}(l)\right)$, where $\operatorname{dec}(\cdot)$ is the decision function which depends on the signalling (or modulation) method. For example, $\operatorname{dec}(x)=\operatorname{sign}(x)$, where $\operatorname{sign}(\cdot)$ is the sign function, if binary phase-shift keying (BPSK) is used.

Suppose that the MMSE criterion is used to find the filter coefficients. Let

$$
\begin{gathered}
\mathbf{g}_{j}=\left[\begin{array}{llll}
g_{j, 0} & g_{j,-1} & \cdots & g_{j,-L_{g}}
\end{array}\right]^{T} \\
u_{j, s,(k)}(l)=\sum_{m=0}^{N-1} y_{j, N l+m-s} c_{(k), N l+m}^{*}, j=1,2, \ldots, L \text { and } \\
v_{t,(k)}(l)=\sum_{m=0}^{N-1} \tilde{x}_{N l+m-t} c_{(k), N l+m}^{*} .
\end{gathered}
$$

In addition, define

$$
\mathbf{u}_{j,(k)}(l)=\left[\begin{array}{llll}
u_{j, 0,(k)}(l) & u_{j,-1,(k)}(l) & \cdots & u_{j,-L_{g},(k)}(l)
\end{array}\right]^{T}
$$

and

$$
\mathbf{v}_{(k)}(l)=\left[\begin{array}{llll}
v_{1,(k)}(l) & v_{2,(k)}(l) & \cdots & v_{L_{h},(k)}(l)
\end{array}\right]^{T} .
$$

From (8) and (9), we can define the symbol-level MSE with the pilot symbol estimate to be

$$
\begin{aligned}
\operatorname{MSE}(\mathbf{g}, \mathbf{v}) & =E\left[\left|\hat{b}_{(1)}(l)-b_{(1)}(l)\right|^{2}\right] \\
& =E\left[\left|\mathbf{g}^{T} \mathbf{u}_{(1)}(l)-\mathbf{f}^{T} \mathbf{v}_{(1)}(l)-b_{(1)}(l)\right|^{2}\right]
\end{aligned}
$$

where $\mathbf{g}=\left[\mathbf{g}_{1}^{T} \mathbf{g}_{1}^{T} \cdots \mathbf{g}_{L}^{T}\right]^{T}, \mathbf{u}_{(1)}(l)=\left[\mathbf{u}_{1,(1)}^{T}(l) \mathbf{u}_{2,(1)}^{T}(l) \cdots\right.$ $\left.\mathbf{u}_{L,(1)}^{T}(l)\right]^{T}$, and $\mathbf{f}=\left[f_{1} f_{2} \cdots f_{L_{h}}\right]^{T}$. The optimal filter vectors conditioned on the given channel coefficients are the vectors which minimize the MSE in (12). The optimal solution of the chip-level MMSE DFE is derived in Appendix A.

Although it seems to be straightforward to employ the chiplevel DFE as shown above, there is a problem: the decided symbols for the $l$ th symbol period in (7) are not available until despreading of the output of the DFE has been performed, as in (9). It naturally leads to an iterative approach which is described in Section IV.

There are some remarks, as follows.

- The chip-level DFE in Fig. 1 can be seen to be a combination of the chip-level LE and the interference canceller [7], [13], [17]. The main difference from the MPI canceller (MPIC) [7] is that only postcursor MPI is subtracted, while all MPI is subtracted in the MPIC. After removing postcursor MPI, linear filtering is used to restore orthogonality and then MAI is suppressed by despreading in the chip-level DFE. Hence, both the linear filter and canceller (which is a nonlinear operation) are used to construct the chip-level DFE. In the MPIC, the performance can be degraded by an incorrect tentative decision. However, in the chip-level DFE, the linear filtering performed by the FFF can prevent the performance degradation from incorrect cancellation in the FBF. From this, the chip-level DFE may provide better performance than the MPIC. See the section on performance results for a comparison.

- The chip-level DFE was also addressed in [21]. For the FBF, the sum of the pilot spread signal and the desired spread signal becomes the input [21]. Hence, it is not required to know all active users, while all active users must be known in the proposed chip-level DFE for the FBF as in (7). Indeed, it is possible to generalize the chip-level DFE with a subset of active users' signals. If the subset consists of the pilot spread signal and the desired spread signal, this chip-level DFE is identical to that in [21]. If the subset becomes the full set, which consists of all active spread signals, the chip-level DFE is the proposed DFE. The optimal solution for this general case can be readily obtained, as shown in Appendix A. 


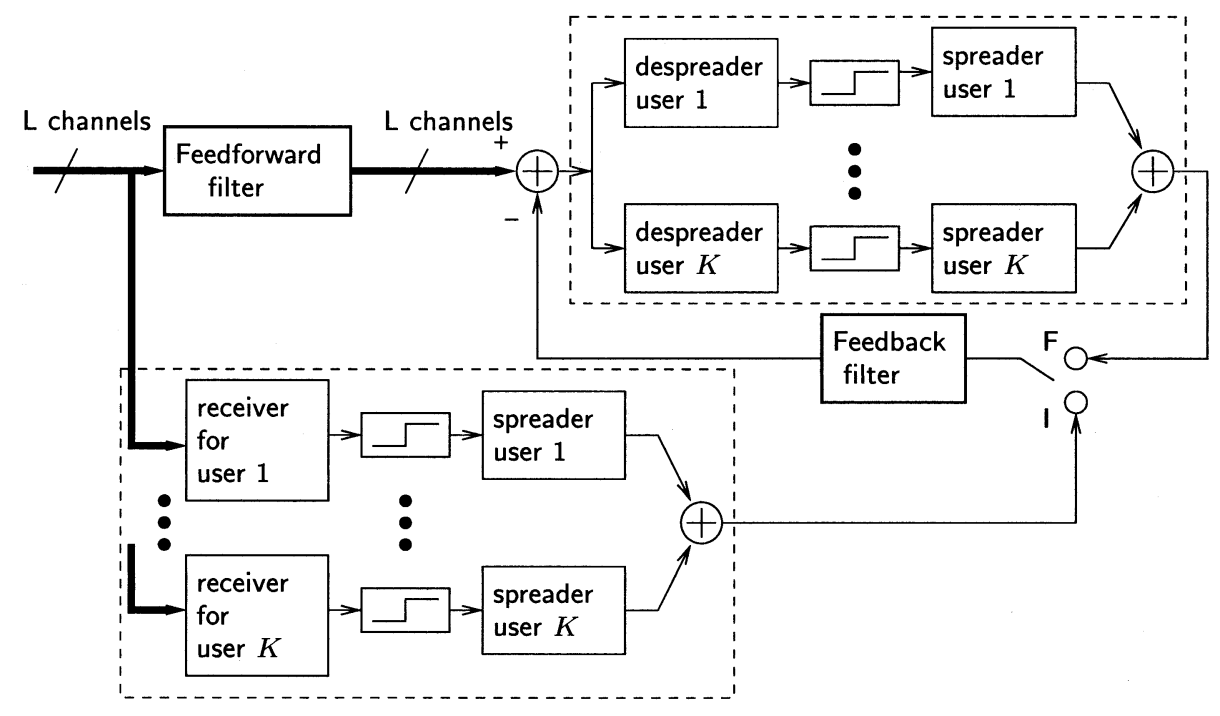

Fig. 2. Block diagram of the (iterative) receiver with chip-level DFE with external receivers for the initial decision.

\section{Proposed Iterative Receivers With ChIP-LeVEl DFE}

In this section, we propose some iterative approaches for the chip-level DFE in a time-variant frequency-selective fading channel. In order to track the variation of the channel, we consider the adaptive chip-level DFE.

\section{A. Hard Decision Based Iterative Receiver With Initial Decision From the Rake Combiners}

As pointed out in Section III, the input of the FBF is not available at first. In order to overcome this problem, a separate external signal detector or receiver can be used for the initial input of the FBF. For example, we can use the rake receiver.

Let the hard decision of the outputs of the external receiver be the initial decision (i.e., the zeroth decision) of the iterative receiver

$$
\tilde{b}_{0,(k)}(l)=\operatorname{dec}\left(\hat{b}_{\mathrm{ext},(k)}(l)\right), \quad k=2,3, \ldots, K
$$

where $\hat{b}_{\text {ext, }(k)}(l)$ denotes the initial decision from the external receiver. The initial decision can be used for the input of the FBF

$$
\begin{aligned}
\tilde{x}_{0, N l+m} & =c_{(1), N l+m} b_{(1)}(l)+\sum_{k=2}^{K} c_{(k), N l+m} \tilde{b}_{0,(k)}(l) \\
m & =0,1, \ldots, N-1 .
\end{aligned}
$$

Then, the output of the DFE is given by

$$
\begin{aligned}
\hat{x}_{1, N l+m}= & \sum_{j=1}^{L} \sum_{p=-L_{g}}^{0} g_{j, p}(l-1) y_{j, N l+m-p} \\
& -\sum_{p=1}^{L_{h}} f_{p}(l-1) \tilde{x}_{0, N l+m-p} \\
m= & 0,1, \ldots, N-1
\end{aligned}
$$

where $\left\{g_{j, p}(l-1)\right\}$ and $\left\{f_{p}(l-1)\right\}$ are the FFF and FBF coefficients obtained from the previous [i.e., the $(l-1)$ th] symbol interval, respectively.
The output of the DFE that is obtained from $\tilde{b}_{0,(k)}(l)$ can be used to find a better decision on $b_{(k)}(l)$ as

$$
\hat{b}_{1,(k)}(l)=\sum_{m=0}^{N-1} \hat{x}_{1, N l+m} c_{(k), N l+m}^{*}, \quad k=2,3, \ldots, K
$$

which can be considered to be the decision on $b_{(k)}(l)$ from the first iteration. This decision also can be fed back through the FBF for the second iteration, and so on. Consequently, we can use this iterative decision for the DFE with the initial decision from the external receiver.

A block diagram of the (iterative) receiver with chip-level DFE when the output of the rake receiver is used for the initial decision is presented in Fig. 2. In the initial mode, the input of the FBF is connected to point I. After the initial decision has been made, the input of the FBF is connected to point $F$ for the feedback mode.

After some iterations, say $Q$, we have the final decision $\hat{b}_{Q,(k)}(l)$. In summary, we have the $q$ th iteration from the $(q-1)$ st iteration given by

$$
\begin{aligned}
\tilde{x}_{q-1, N l+m}= & c_{(1), N l+m} b_{(1)}(l) \\
& +\sum_{k=2}^{K} c_{(k), N l+m} \tilde{b}_{q-1,(k)}(l) \\
m= & 0,1, \ldots, N-1 \\
\hat{x}_{q, N l+m}= & \sum_{j=1}^{L} \sum_{p=-L_{g}}^{0} g_{j, p}(l-1) y_{j, N l+m-p} \\
& -\sum_{p=1}^{L_{h}} f_{p}(l-1) \tilde{x}_{q-1, N l+m-p} \\
m= & 0,1, \ldots, N-1 \\
\tilde{b}_{q,(k)}(l)= & \operatorname{dec}\left(\sum_{m=0}^{N-1} \hat{x}_{q, N l+m} c_{(k), N l+m}^{*}\right) \\
k= & 2,3, \ldots, K .
\end{aligned}
$$


After the symbols are finally decided, the FFF and FBF should be updated to minimize the MSE in (12). Let

$$
\tilde{v}_{p,(k)}(l)=\sum_{m=0}^{N-1} \tilde{x}_{Q, N l+m-p} c_{(k), N l+m}^{*}
$$

and

$$
\tilde{\mathbf{v}}_{(k)}(l)=\left[\begin{array}{llll}
\tilde{v}_{1,(k)}(l) & \tilde{v}_{2,(k)}(l) & \cdots & \tilde{v}_{L_{h},(k)}(l)
\end{array}\right]^{T} .
$$

The recursive least squares (RLS) algorithm [6] can be used to update $\mathbf{g}$ and $\mathbf{f}$ to minimize the exponentially weighted error from (12), namely

$$
\mathcal{E}(l)=\sum_{i=-\infty}^{l} \lambda^{l-i}\left|e_{(1)}(i)\right|^{2}
$$

where $\lambda$ is the forgetting factor and $e_{(1)}(i)$ is the error between the pilot symbol and its estimate obtained after $Q$ iterations and given by $e_{(1)}(i)=\mathbf{g}^{T}(i) \mathbf{u}_{(1)}(i)-\mathbf{f}^{T}(i) \tilde{\mathbf{v}}_{(1)}(i)-b_{(1)}(i)$. The RLS algorithm is summarized as

$$
\begin{aligned}
\mathbf{k}(l) & =\frac{\mathbf{P}(l-1) \mathbf{d}(l)}{\lambda+\mathbf{d}^{H}(l) \mathbf{P}(l-1) \mathbf{d}(l)} \\
\alpha(l) & =b_{(1)}(l)-\mathbf{w}^{H}(l) \mathbf{d}(l) \\
\mathbf{w}(l) & =\mathbf{w}(l-1)+\mathbf{k}(l) \alpha^{*}(l) \\
\mathbf{P}(l) & =\lambda^{-1} \mathbf{P}(l-1)-\lambda^{-1} \mathbf{k}(l) \mathbf{d}^{H}(l) \mathbf{P}(l-1)
\end{aligned}
$$

where $\mathbf{k}(l)$ is the gain vector, $\alpha(l)$ is the innovation, $\mathbf{P}(l)$ is the inverse of the correlation matrix, and

$$
\mathbf{w}(l)=\left[\begin{array}{ll}
\mathbf{g}^{T}(l) & \mathbf{f}^{T}(l)
\end{array}\right]^{T} \text { and } \mathbf{d}(l)=\left[\mathbf{u}_{(1)}^{T}(l)-\tilde{\mathbf{v}}_{(1)}^{T}(l)\right]^{T} .
$$

Since the MSE in (12) is a quadratic function of $\mathbf{w}(l)$, the RLS algorithm in (20) is the standard one and most convergence properties can be found in [6]. It would be desirable to see the tracking performance for time-variant fading channels. However, it is beyond the scope of the paper and we do not pursue it.

\section{B. Soft Decision Based Iterative Receiver}

Suppose that the equalization is ideal or close to ideal. Then, MAI can be ignored after despreading. By ignoring the correlation of the noise components of $\hat{b}_{q,(k)}(l), k=2,3, \ldots, K$, and given that the previous decision is correct, the joint probability density function (pdf) can be approximated as

$$
\begin{aligned}
f\left(\hat{\mathbf{b}}_{q}(l) \mid \hat{\mathbf{b}}_{q-1}(l)\right. & =\mathbf{b}(l)) \\
& \simeq C \exp \left(-\frac{1}{\sigma_{w}^{2}}\left\|\hat{\mathbf{b}}_{q}(l)-\mathbf{b}(l)\right\|^{2}\right)
\end{aligned}
$$

where $C$ is a constant, $\hat{\mathbf{b}}(l)=\left[\hat{b}_{(2)}(l) \hat{b}_{(3)}(l) \ldots \hat{b}_{(K)}(l)\right]^{T}$, $\mathbf{b}(l)=\left[b_{(2)}(l) b_{(3)}(l) \ldots b_{(K)}(l)\right]^{T}$, and $\sigma_{w}^{2}$ is the noise variance of $w_{N l+m}$. From (8) and (9), it can be shown that

$$
E\left[\left|w_{N l+m} c_{(1), N l+m}^{*}\right|^{2}\right]=\sigma_{w}^{2}
$$

because $c_{(k), N l+m}$ are normalized. Based on this pdf, we can obtain soft decision in our iteration process. Let $b_{(k)}(l) \in \mathcal{S}=$ $\left\{s_{1}, s_{2}, \ldots, s_{P}\right\}$, where the $s_{p}$ s represent the data symbols and $P$ is the size of the symbol alphabet. By assuming that the $b_{(k)}(l)$ $s$ are equally likely and independent of each other, the a posteriori probability that $b_{q,(k)}(l)=s_{p}$ is given by

$$
\begin{aligned}
P_{q,(k)}(l ; p)= & \operatorname{Pr}\left(b_{q,(k)}(l)=s_{p} \mid \hat{b}_{q,(k)}(l), \hat{\mathbf{b}}_{q-1}(l)=\mathbf{b}(l)\right) \\
\propto & f\left(\hat{b}_{q,(k)}(l) \mid b_{(k)}(l)=s_{p}, \hat{\mathbf{b}}_{q-1}(l)=\mathbf{b}(l)\right) \\
& \times \operatorname{Pr}\left(b_{(k)}(l)=s_{p}\right) \\
\propto & \propto\left(\hat{b}_{q,(k)}(l) \mid b_{(k)}(l)=s_{p}, \hat{\mathbf{b}}_{q-1}(l)=\mathbf{b}(l)\right) .
\end{aligned}
$$

Hence, using (22), we can show that

$$
P_{q,(k)}(l ; p) \simeq \frac{\exp \left(-\frac{1}{\sigma_{w}^{2}} \mathcal{M}\left(\hat{b}_{q,(k)}(l), s_{p}\right)\right)}{\sum_{p^{\prime}=1}^{P} \exp \left(-\frac{1}{\sigma_{w}^{2}} \mathcal{M}\left(\hat{b}_{q,(k)}(l), s_{p^{\prime}}\right)\right)}
$$

where $\mathcal{M}\left(\hat{b}_{q,(k)}(l), s_{p}\right)=\left|\hat{b}_{q,(k)}(l)-s_{p}\right|^{2}$ is the metric. As in iterative decoding [5], if an extrinsic information is available, the a priori probability $\operatorname{Pr}\left(b_{(k)}(l)=s_{p}\right)$ in (23) can be changed or updated through iteration. Although the iteration including the channel decoding for coded sequences can provide better performance, it may significantly increase the complexity of the receiver of the mobile station. Hence, we do not discuss it in the paper.

Note that in (24) the variance $\sigma_{w}^{2}$ is known. For each iteration, an estimate of $\sigma_{w}^{2}$ can be given by

$$
\begin{aligned}
\hat{\sigma}_{q, w}^{2}(l)=\frac{1}{K}\left(\mid \hat{b}_{q,(1)}(l)\right. & -\left.b_{(1)}(l)\right|^{2} \\
& \left.+\sum_{k=2}^{K} \min _{s_{p} \in \mathcal{S}}\left|\hat{b}_{q,(k)}(l)-s_{p}\right|^{2}\right) .
\end{aligned}
$$

This estimate of $\sigma_{w}^{2}$ is obtained by assuming that $\tilde{s}_{p}=\arg \min _{s_{p} \in \mathcal{S}}\left|\hat{b}_{q,(k)}(l)-s_{p}\right|^{2}$ is the correct decision of $b_{(k)}(l)$ for $k=2,3, \ldots, K$.

Using $P_{q,(k)}(l ; p)$ in (24), we can have a soft decision for each iteration as

$$
\bar{b}_{q,(k)}(l)=\sum_{p=1}^{P} P_{q,(k)}(l ; p) s_{p}, \quad k=2,3, \ldots, K .
$$

With this soft decision, the iteration in Section IV-A can be carried out. We can observe a minor performance improvement through computer simulations (see Section IV) by using the soft decision. However, there is another advantage of this soft decision. With the metrics or the a posteriori probabilities in (24), we can find some data symbols that are reliably decided. These decided symbols can be used as pilot signals (or known symbols) to update the FFF and FBF further, to improve the tracking performance as follows.

After $Q$ iterations, according to (24), a symbol, say $\hat{b}_{Q,(k)}(l)$, can be decided with high reliability if $P_{Q,(k)}(l ; p)$ is high or $\mathcal{M}\left(\hat{b}_{q,(k)}(l), s_{p}\right)$ is small for some $p$. Let $\bar{K}$ be the number of the decided symbols with high reliability. Define the reliability measure from the metric as

$$
R\left(\hat{b}_{q,(k)}(l)\right)=\min _{s_{p} \in \mathcal{S}}\left\{\mathcal{M}\left(\hat{b}_{q,(k)}(l), s_{p}\right)\right\}, \quad k=2,3, \ldots, K .
$$

Then, the decided symbols corresponding to the $\bar{K}$ smallest reliability measures among $R\left(\hat{b}_{Q,(k)}(l)\right), k=2,3, \ldots, K$, can 
TABLE I

LIST OF PARAMETERS FOR SIMULATIONS

\begin{tabular}{c|c}
\hline Parameter & Value \\
\hline \hline Carrier frequency & $2 \mathrm{GHz}$ \\
\hline Modulation & QPSK \\
\hline Data rate & $64 \mathrm{Kbps}$ \\
\hline Processing gain & 32 \\
\hline Spreading code & Scrambled Walsh codes \\
\hline Root mean square delay spread & $5 \mu$ sec. \\
\hline Number of multipaths, $L_{h}+1$ & 6 \\
\hline Length of FFF of DFE, $L_{g}+1$ & 8 \\
\hline Length of LE, $L_{g}+1$ & 8 \\
\hline Smoothing factor of channel estimation for the rake receiver, $B$ & 50 \\
\hline
\end{tabular}

be chosen for further updating [note that, as $R(\cdot)$ has smaller value, it has more reliability]. Denote by $\mathcal{I}_{R}$ the index set for the $\bar{K}$ decided symbols with high reliability. That is, $\mathcal{I}_{R}=$ $\left\{k_{1}, k_{2}, \ldots, k_{\bar{K}}\right\}$, where $R\left(\hat{b}_{Q,\left(k_{p}\right)}(l)\right) \leq R\left(\hat{b}_{Q,\left(k_{p+1}\right)}(l)\right)$ and $R\left(\hat{b}_{Q,\left(k_{p+1}\right)}(l)\right) \leq R\left(\hat{b}_{Q,(k)}(l)\right)$ for $k \notin \mathcal{I}_{R}$. Since we have $(\bar{K}+1)$ pilot symbols at the lth symbol interval, the exponentially weighted error for the RLS algorithm is defined with $(\bar{K}+1)$ errors to be

$$
\mathcal{E}(l)=\sum_{i=1}^{l} \lambda^{l-i}\left(\left|e_{(1)}(i)\right|^{2}+\sum_{p=1}^{\bar{K}}\left|e_{\left(k_{p}\right)}(i)\right|^{2}\right)
$$

where $e_{(k)}(i)=\mathbf{g}^{T}(i) \mathbf{u}_{(k)}(i)-\mathbf{f}^{T}(i) \tilde{\mathbf{v}}_{(k)}(i)-\operatorname{dec}\left(\hat{b}_{Q,(k)}(i)\right)$. Unfortunately, in this case, computationally efficient implementation of the RLS algorithm in (20) is not available, because the matrix inversion lemma is not applicable to update the inverse of the correlation matrix.

In order to use the RLS algorithm in (20), we need to modify the weighted error function

$$
\mathcal{E}(l)=\sum_{i=-\infty}^{l} \lambda^{l-i}\left(\left|e_{(1)}(i)\right|^{2}+\sum_{p=1}^{\bar{K}} \beta^{p}\left|e_{\left(k_{p}\right)}(i)\right|^{2}\right)
$$

where $\beta$ is a weighting factor. Since $R\left(\hat{b}_{Q,\left(k_{p}\right)}(l)\right) \leq$ $R\left(\hat{b}_{Q,\left(k_{p+1}\right)}(l)\right)$, the decision for $\hat{b}_{Q,\left(k_{p}\right)}(l)$ is more reliable than for $b_{Q,\left(k_{p+1}\right)}(l)$. From this, $\beta$ should be less than one to reflect this reliability weighting. In addition, if we let

$$
\beta=\lambda^{1 /(\bar{K}+1)}
$$

the RLS algorithm can be directly utilized without any modification. In order to show this, define the following new error sequence:

$$
\bar{e}((\bar{K}+1) i+p)= \begin{cases}e_{\left(k_{\bar{K}-p}\right)}(i), & p=0,1, \ldots, \bar{K}-1 \\ e_{(1)}(i), & p=\bar{K} .\end{cases}
$$

Let $m=(\bar{K}+1) i+p$. Then, the weighted error function in (27) is rewritten as

$$
\mathcal{E}(m)=\sum_{i=-\infty}^{m} \beta^{m-i}|\bar{e}(i)|^{2} .
$$

The RLS algorithm with (30) is equivalent to the RLS algorithm which updates the filter vectors $(\bar{K}+1)$ times per symbol with the order that $e_{\left(k_{\bar{K}}\right)}(i)$ is the first, $e_{\left(k_{\bar{K}-1}\right)}(i)$ the second, and so on, until $e_{(1)}(i)$ the last. Clearly, it increases the burden of computation by $(\bar{K}+1)$ times for updating the DFE filters.
However, it would be effective when the variation of the channel is fast, because the filter vectors can be updated more often to track the variation of the channel.

\section{Simulation Results}

In Table I, the parameters used for simulations are listed. The time-variant complex channel coefficients have the following autocorrelation

$$
E\left[h_{j, p ; l} h_{j, p^{\prime} ; l^{\prime}}^{*}\right]=\sigma_{j, h ; p}^{2} J_{0}\left(2 \pi f_{D} T_{s}\left|l-l^{\prime}\right|\right) \delta_{p, p^{\prime}} \delta_{j, j^{\prime}}
$$

where $T_{s}$ is the symbol interval, $f_{D}$ is the maximum Doppler frequency, and $J_{0}(\cdot)$ is the zeroth order Bessel function of the first kind. The variance of the channel coefficients is normalized by

$$
\sum_{p=0}^{L_{h}} \sigma_{j, h ; p}^{2}=1, \text { for all } j=1,2, \ldots, L
$$

and each CIR has an exponential power delay profile with a root mean-square (rms) delay spread of $5 \mu \mathrm{s}$, as in Table I. Since we use the quadrature phase-shift keying (QPSK), the signal set becomes $\mathcal{S}=\{ \pm 1 \pm i\}$ and $P=4$. Together with (31), this means that the received SNR is given by SNR $=E_{b} / N_{0}=$ $1 / N_{0}$, where $E_{b}$ is the bit energy. In simulations, we consider the three different receivers: the rake receiver, the receiver with chip-level LE, and the receiver with chip-level DFE (both the hard and soft decisions are considered).

\section{A. Comparison of Rake Receiver, LE, and DFE With Known Channel}

Since the BER performance depends on channel coefficients, it would be desirable to obtain the BER averaged over the channel coefficients. However, it is not easy to find a closed-form expression for the BER of the DFE, although the closed-form solutions of the optimal LE and DFE are available. Hence, in this subsection, we present the simulation results of the BER. It is assumed that the channel coefficients are known so that the optimal LE and DFE are computed. The optimal MMSE DFE is found in Appendix A. Furthermore, it is assumed that the feedback is correct in the DFE (i.e., there is no error propagation).

In Fig. 3, the BER curves are shown in terms of the SNR (in decibels) when the system is half loaded $(K=17)$. It is shown that the chip-level equalizers can provide much better performance than the rake receiver as the SNR gets higher. When $L=1$, the error 


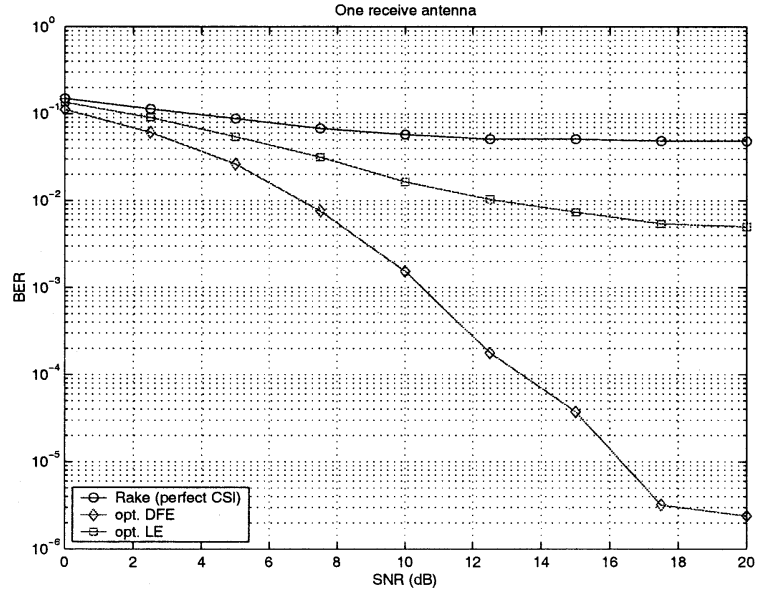

(a)

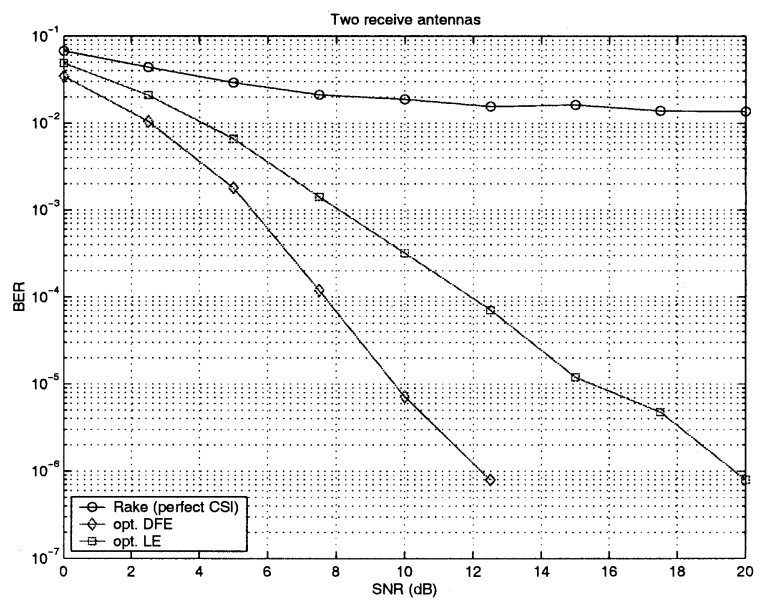

(b)

Fig. 3. BER versus SNR (in decibels) $(K=17)$.

flooring effect is observed, with the chip-level LE as shown in Fig. 3(a), while it is not observed with the chip-level DFE. It is due to the residual MAI and MPI, since the LE cannot perfectly equalize the channel with a finite number of taps. However, if there is more than one receiving antenna, it is possible to equalize the channel perfectly. Hence, there is no error flooring effect, as shown in Fig. 3(b). Although the LE performs better than the rake receiver, the performance of the LE generally is worse than that of the DFE. When $L=2$, the LE requires a 3-dB larger SNR than the DFE to achieve a BER of $10^{-3}$. As the target BER gets lower, a higher SNR is required with the LE.

In the proposed chip-level DFE, all the active signals are used for the FBF. This requires the identification of active users or spreading codes. Although it is not difficult to identify (or detect) active users, it increases the complexity of the receiver. Therefore, it is important to see the performance improvement when including all active users for the FBF. We assume that there are 20 active users and consider the case when $K_{s} \leq 20$ active users' spreading signals are used for the FBF. If $K_{s}=$ 2 (the desired user's spread signal and the pilot signal), this chip-level DFE is identical to that in [21]. The BER results are shown in Fig. 4. It is shown that if more active spread signals are included, the performance is improved. When $K_{s}=2$, the performance of the DFE is slightly better than that of the LE. It

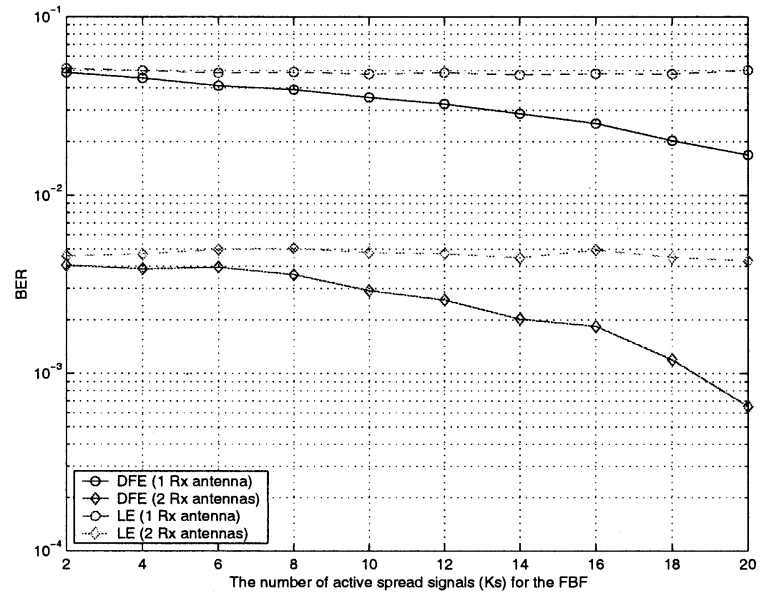

Fig. 4. BER performance for different number of active spread signals that are taken into account for the FBF $(\mathrm{SNR}=6 \mathrm{~dB}$ and $K=20$ ).

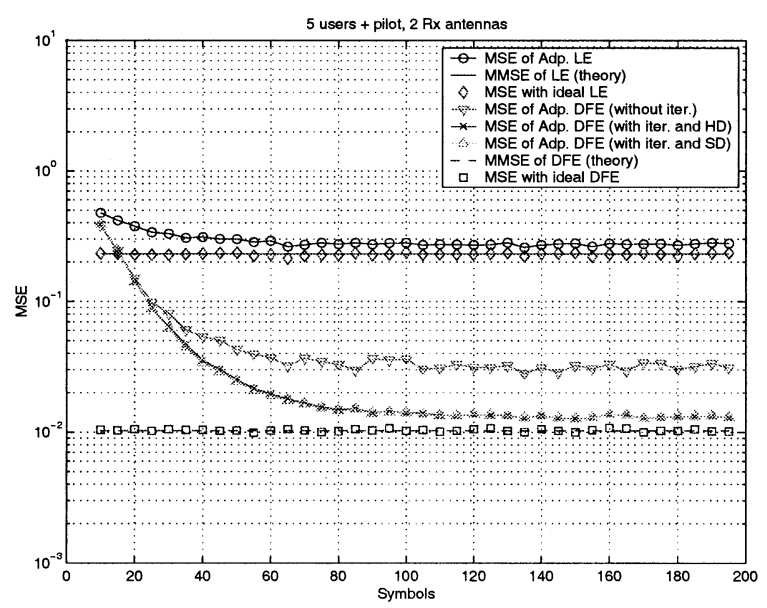

Fig. 5. MSE curves $(L=2$, SNR $=20 \mathrm{~dB}$, and $K=6)$.

shows that all active users' spread signals should be taken into account for better performance.

In order to see convergence properties, we consider static channels in this subsection. It is assumed that two receiving antennas are used and the CIRs are as follows:

$$
\begin{aligned}
& \left\{h_{1,0}, h_{1,1}, \ldots, h_{1,5}\right\} \\
& =\{0.5070,0.4598,0.4171,0.3782,0.3431,0.3111\} \\
& \left\{h_{2,0}, h_{2,1}, \ldots, h_{2,5}\right\} \\
& =\{0.5070,-0.4598,0.4171,-0.3782,0.3431,-0.3111\} .
\end{aligned}
$$

Three different types of receiver with chip-level DFE have been considered: the first is with one iteration, i.e., $Q=1$ (Type 1), the second is with more than one iterations and hard decision for feedback (Type 2), and the third is with more than one iterations and soft decision for feedback (Type 3 ). All three receivers use the rake receiver for the initial decision. The number of iterations $Q$ is set to three in Types 2 and 3 . A code-multiplexed pilot is used to update the filter coefficients in Types 1-3. In Type 3, only a codemultiplexed pilot is utilized for adaptation (i.e., $\bar{K}=0$ ).

With $\lambda=0.98$, the MSE curves are shown in Fig. 5. The adaptive chip-level LE is also considered with the RLS algorithm. As shown in Fig. 5, the theoretical MMSE solution derived in Appendix A is in good agreement with simulation results. In the adaptive chip-level DFE, it is shown that iterations 


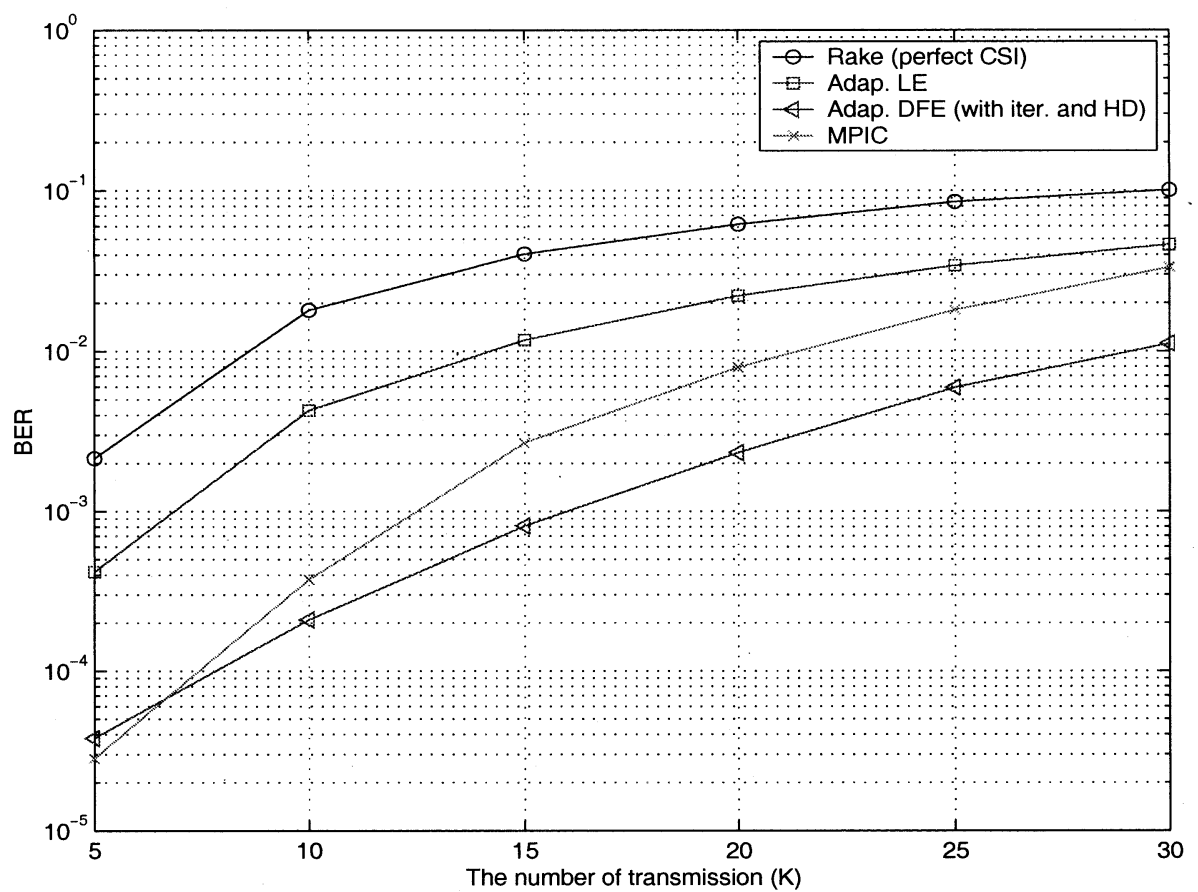

(a)

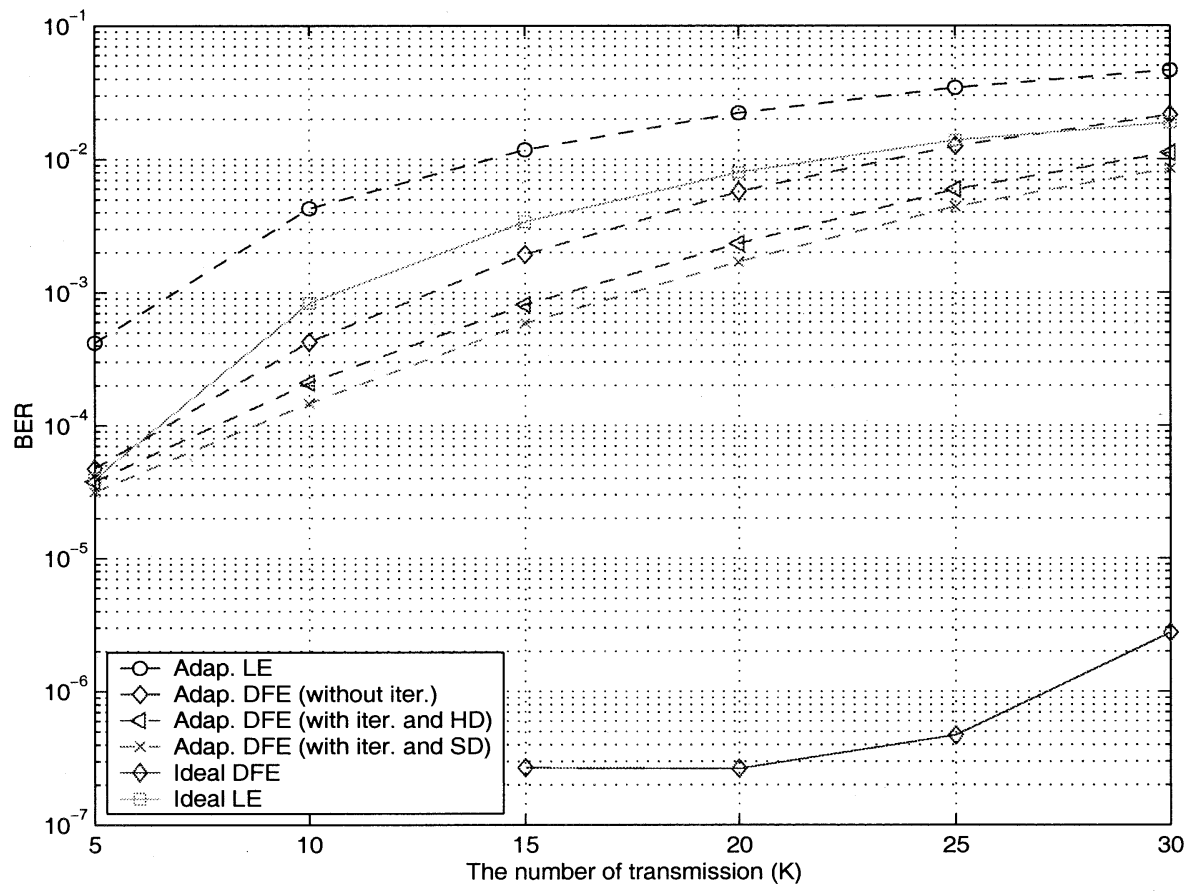

(b)

Fig. 6. BER versus number of users, $K$ (Mobile speed $=60 \mathrm{~km} / \mathrm{hr}, \mathrm{SNR}=20 \mathrm{~dB}, \lambda=0.95$, and $Q=2$ ).

help to improve the performance (Types 2 and 3 perform better than Type 1). Note that there exists the excess MSE in both adaptive LE and DFE [6]. Hence, there is always a difference between the actual MSE and MMSE.

\section{B. Results of Adaptive Chip-Level Equalizers and MPIC}

In this section, we focus on the performance of an adaptive chip-level equalizer with a single receiving antenna for a time-variant channel. A code-multiplexed pilot is used to up- date the filter coefficients of LE and DFE. For comparison, we consider the MPIC in [7] with the channel estimation in (5). As in [3], the outputs of the chip-level LEs are used for the initial symbol decision for the MPIC and the number of iterations for the MPIC is set to one. The chip-level DFE has also the same initial decision as the MPIC for fair comparison.

In Fig. 6, the BER performance of the receivers is shown in terms of the number of transmissions, $K$, including a code-multiplexed pilot. The forgetting factor of the RLS for the adaptive chip-level equalizers, $\lambda$, is set to 0.95 . The number of iterations 


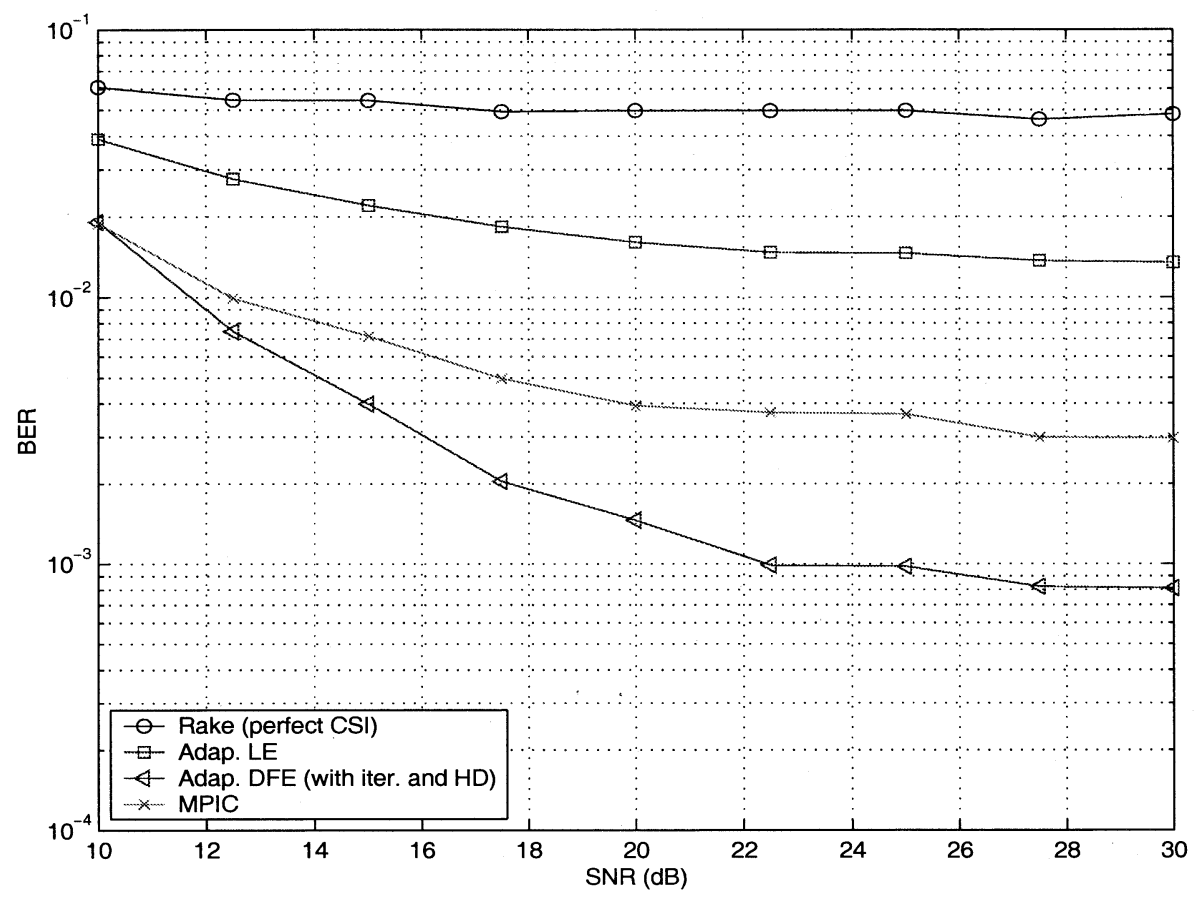

(a)

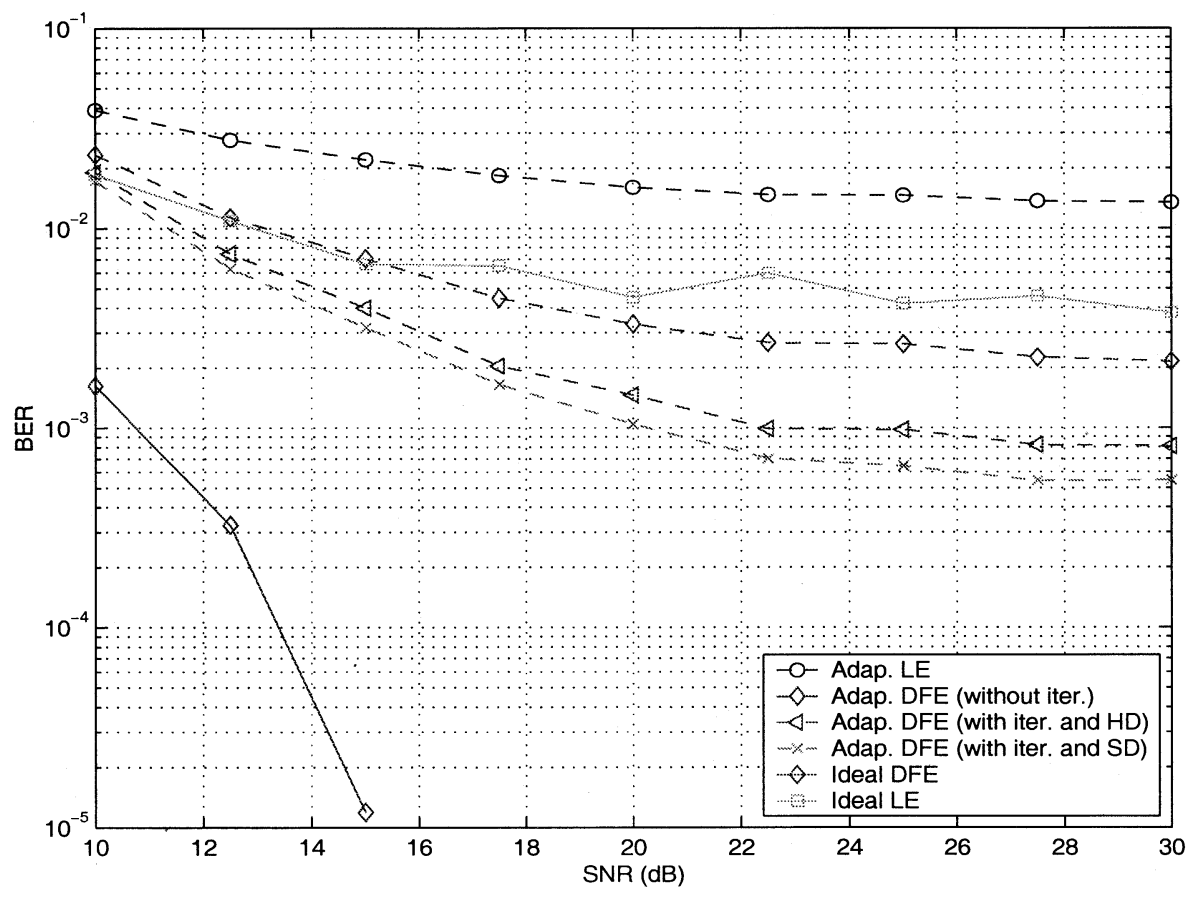

(b)

Fig. 7. BER versus SNR (in decibels) (Mobile speed $=60 \mathrm{~km} / \mathrm{hr}, K=17, \lambda=0.95$, and $Q=2$ ).

$Q$ is set to 3 and the filter vectors are updated with a code-multiplexed pilot only (i.e., $\bar{K}=0$ in Type 3 ). We can see that the receiver with chip-level equalizers can perform better than the rake receiver, even if the rake receiver uses the perfect channel state information (CSI) (i.e., the case that the channel coefficients are perfectly known by the rake receiver). Generally, the chip-level DFE performs better than the chip-level LE and the MPIC [see Fig. 6(a)]. The MPIC only can provide better performance than the chip-level DFE when the system load is small $(K=5)$. Since the performance of the MPIC depends on the interference cancellation, the correct decision is crucial. As $K$ gets large, there are more incorrectly decided symbols and the performance degrades. On the other hand, the performance of the chip-level DFE depends on both the linear filtering by the FFF and the interference cancellation by the FBF. Even though some decisions are incorrectly made, the FFF can prevent performance degradation from incorrect cancellation. In addition, in the adaptive chip-level DFE, we can see that the iteration can help to improve the performance significantly and the soft decision is better than the hard decision [see Fig. 6(b)]. 


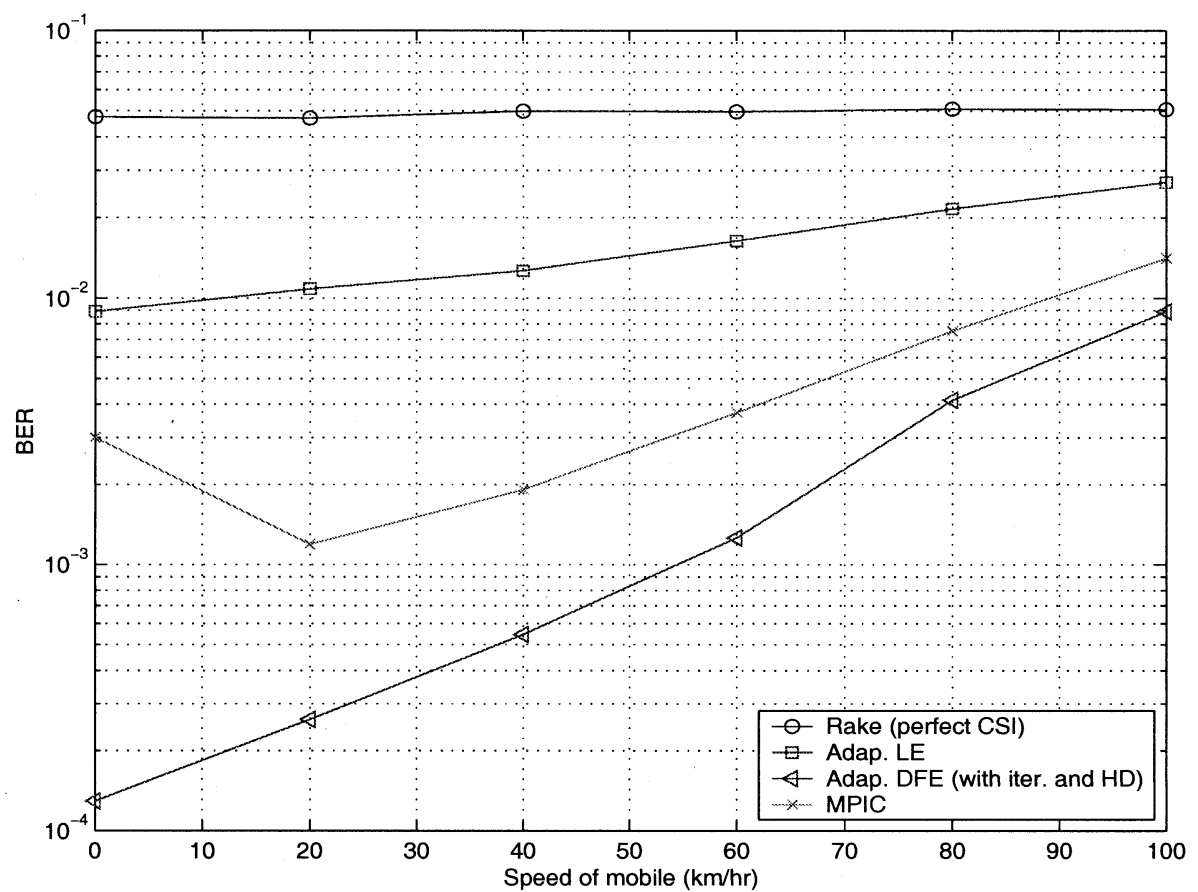

(a)

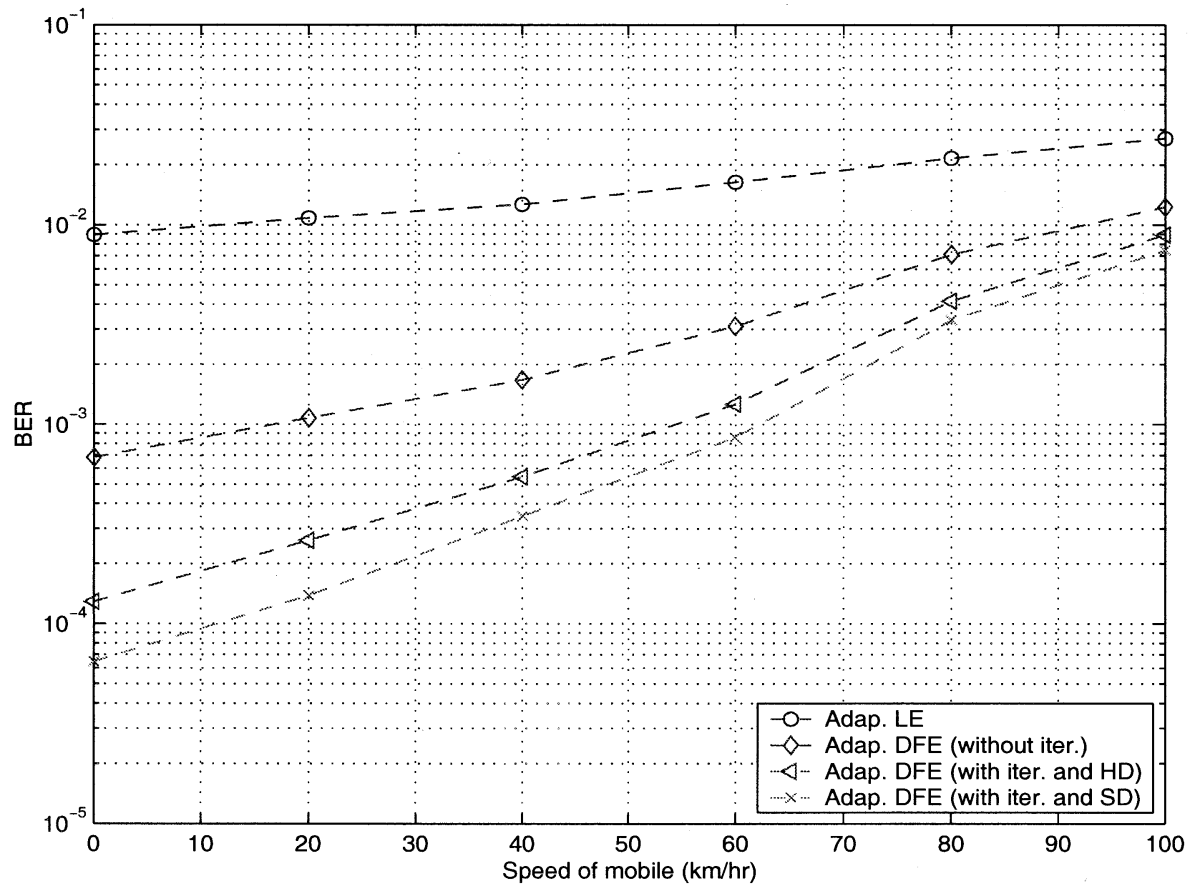

(b)

Fig. 8. BER versus speed of mobile ( $\mathrm{SNR}=20 \mathrm{~dB}, K=17, \lambda=0.95$, and $Q=2$ ).

There is a large performance gap between ideal chip-level DFE and adaptive chip-level DFE, as shown in Fig. 6(b). One reason is that there exist decision errors in feedback in the adaptive chip-level DFE (in the ideal chip-level DFE, we assume that all decisions are correct and there is no error propagation). Another reason is that the filter coefficients are not perfectly estimated due to the variation of the channel (it may be the dominant factor degrading the performance). These degrade performance and the result will be worse than in the ideal case.
The BER performance in terms of SNR is shown in Fig. 7 when the CDMA system is half-loaded $(K=17)$ and the speed of the mobile is $60 \mathrm{~km} / \mathrm{hr}$. The number of iterations $Q$ is set to $3, \lambda=0.95$ and the filter vectors are updated with a code-multiplexed pilot only (i.e., $\bar{K}=0$ in Type 3 ). Since the performance of the rake receiver strongly depends on the level of MAI, it is not significantly improved as the SNR gets higher. The MPIC can provide better performance than the chip-level LE [see Fig. 7(a)]. Since the receivers of Types 1, 2, and 3 can reduce MAI, these BERs decrease with SNR [see Fig. 7(b)]. How- 


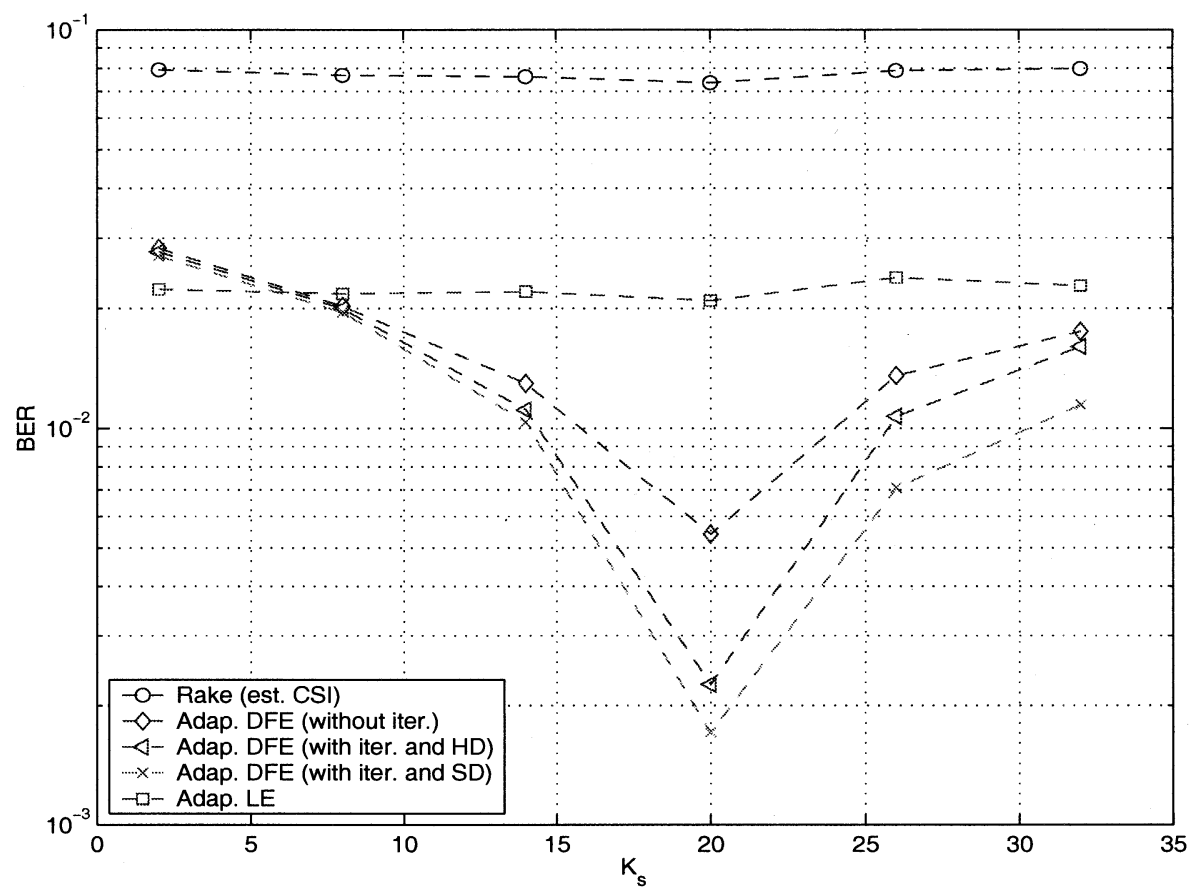

Fig. 9. The impact of wrongly detected inactive and active users on BER performance (SNR $=20 \mathrm{~dB}, K=20$, Mobile speed $=60 \mathrm{~km} / \mathrm{hr}, \lambda=0.95$, and $Q=2$ ).

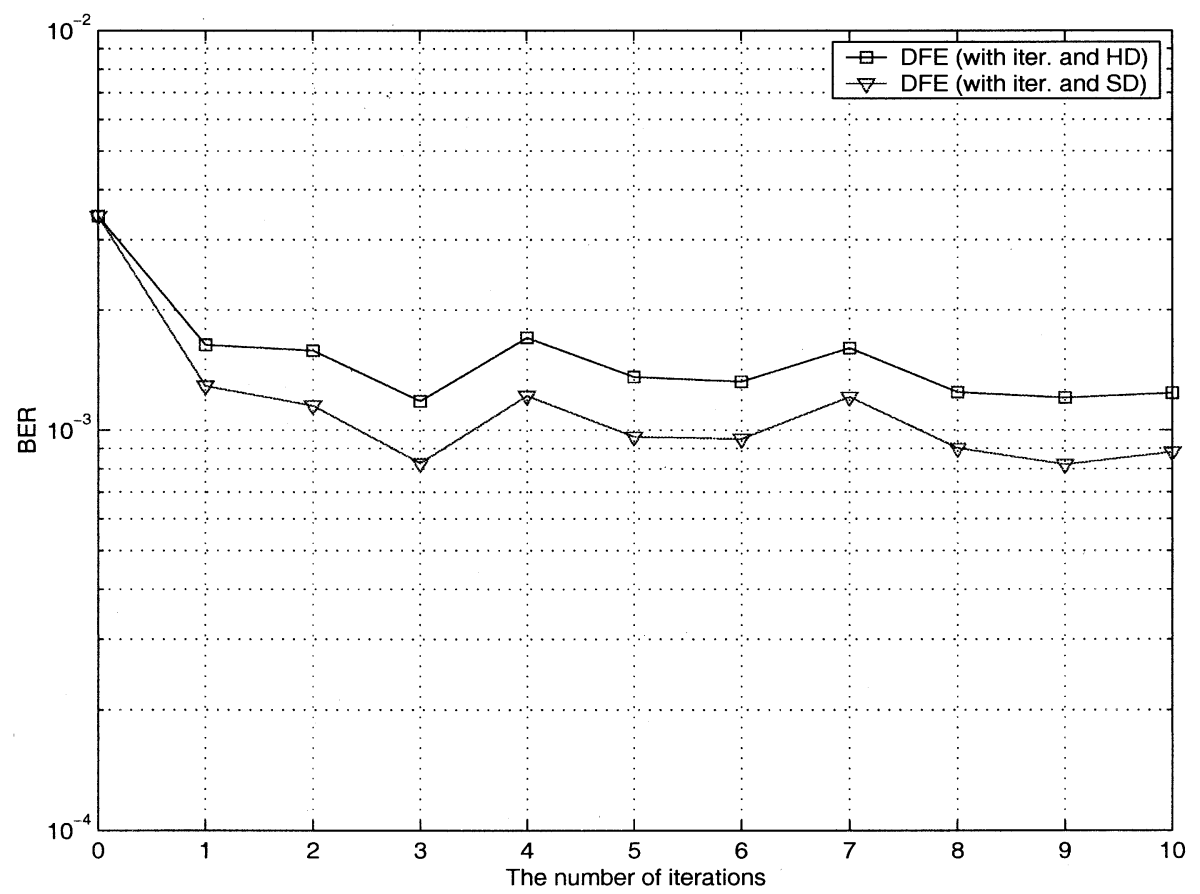

Fig. 10. BER versus number of iteration, $Q$ (Mobile speed $=60 \mathrm{~km} / \mathrm{hr}, K=17$, SNR $=20 \mathrm{~dB}$, and $\lambda=0.95$ ).

ever, all receivers have the error flooring effect at high SNR. This mainly results from estimation errors of filter coefficients in LE and DFE.

Since an adaptive algorithm is used to find the filter coefficients of the FFF and FBF, the performance is affected by the speed of mobile. In Fig. 8, the BER performance in terms of mobile speed is presented for the case when the system is half-loaded and the SNR is $20 \mathrm{~dB}$. With a high-speed mobile, we certainly need to have better adaptation algorithms to decrease tracking errors. For better performance, the Kalman filter can be used to track the channel coefficients, and then the coefficients of the FFF and FBF can be found using the optimal MMSE solution in Appendix A through the estimated channel coefficients. Although it may demand high computational complexity, this should be investigated in the future.

We have assumed that active users are known throughout this paper. Since active users have to be detected in practice, some inactive users may be wrongly regarded as active users, and vice versa. To see the impact of wrongly identified inactive and active users, define the set of indices of detected active users as $\mathcal{K}_{\text {det }}=$ 


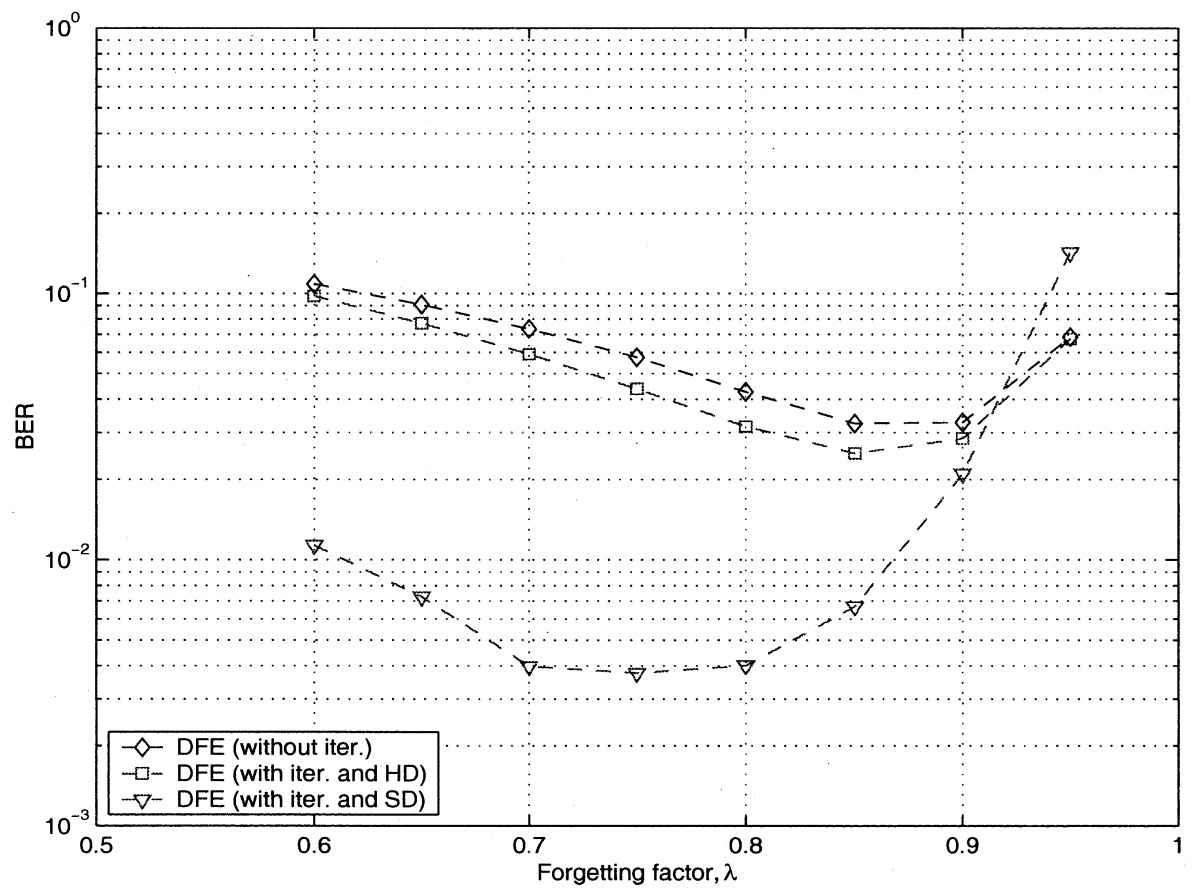

Fig. 11. BER versus forgetting factor, $\lambda$ (Mobile speed $=200 \mathrm{~km} / \mathrm{hr}, K=17, Q=2$, and $\mathrm{SNR}=20 \mathrm{~dB}$ ).

$\left\{1,2, \ldots, K_{s}\right\}$, while the set of indices of active users is $\mathcal{K}=$ $\{1,2, \ldots, 20\}$. Hence, if $K_{s}=20$, then the detection of all the active users is correct. In the case of $K_{s}<20$, there exist active users who are not taken into account. On the other hand, if $K_{s}>20$, there are inactive users who are regarded as active. In both cases, performance degradation is expected because of errors in the feedback of the DFE. The BER results are shown in Fig. 9. As shown, the best performance can be achieved when $K_{s}=K=20$. It is noteworthy that when $K_{s}=2$ (i.e., the receiver knows the spreading codes of the desired user and pilot only), the performance is not better than that of adaptive LE. In the ideal case (with the optimal weight vectors for the FFF and FBF), however, the chip-level DFE performs slightly better than the chip-level LE (see Fig. 4). This shows that, when the weight vectors for the FFF and FBF are estimated through an adaptive algorithm, the chip-level DFE cannot perform better than the chiplevel LE unless the chip-level DFE has reasonably small feedback errors (when $K=20, K_{s}=2$ causes large feedback errors).

In the receivers of Types 2 and 3, the number of iterations, $Q$, is important. The BER may decrease with $Q$. In Fig. 10, the BER has been obtained for different numbers of iterations, with the system being half-loaded $(K=17)$ and the SNR $20 \mathrm{~dB}$. The forgetting factor of the RLS, $\lambda$, is set to 0.95 . The more iterations, the better the performance. However, this increases the burden of computation. In most cases, we find that two or three iterations can provide reasonably improved performance.

When the speed of the mobile is high, the variation of the channels becomes fast. In this case, the adaptation of the filter vectors for the chip-level DFE could make it difficult to track the variation of the channel, as shown in Fig. 8. However, if we can update the filter vectors more than once per symbol interval, the performance of the adaptive chip-level DFE would be better. The use of reliably decided symbols to update the filter vectors was discussed in Section IV-B. In Fig. 11, we present the
BER performance for different values of the forgetting factor, $\lambda$, when the speed of the mobile is $200 \mathrm{~km} / \mathrm{hr}$, which is a reasonably high speed, and its corresponding maximum Doppler frequency is $370.37 \mathrm{~Hz}$. The receiver of Type 3 uses two reliably decided symbols (i.e., $\bar{K}=2$ ) to update the filter vectors of the chip-level DFE. This shows that the performance of the receiver of Type 3 can be better and results in better performance of the chip-level DFE to track the variation of the channel, at the expense of increased computational complexity.

\section{CONCLUDING REMARKS}

In the paper, we have investigated the receiver with chiplevel MMSE DFE for CDMA downlink when the spreading sequences are orthogonal. The MMSE solution has been derived in a closed-form. The chip-level DFE can provide better performance than the chip-level LE and MPIC (and much better performance than the rake receiver).

Due to the inherent structure of the chip-level DFE, iterative receivers have been proposed with reasonable complexity. It is shown that the performance can be significantly improved by iteration. For the feedback in DFE, hard decision and soft decision have been considered. It is shown that soft decision can provide better performance with the additional advantage that since some symbols can be decided with high reliability, these can be used as pilot symbol to update the filter vectors of the DFE. This improves the performance of the chip-level DFE when the variation of the channel is fast. As a result, better BER performance is achieved.

\section{APPENDIX}

\section{Closed-Form MMSE SOLUTION FOR CHIP-LEVEl DFE}

In this Appendix, we derive the MMSE solution for the chiplevel DFE. To find the solution, we assume that the channel 
coefficients are constants and feedback is correct (i.e., there is no propagation error). The spreading sequences are assumed to be the following:

A1) orthogonal to each other in a symbol interval [see (1)];

A2) independent and identically distributed (iid);

$$
E\left[c_{(k), n} c_{\left(k^{\prime}\right), n^{\prime}}^{*}\right]=\frac{1}{N} \delta_{k, k^{\prime}} \delta_{n, n^{\prime}}
$$

and the symbols are iid and equally likely, $L_{g} \geq L_{h}$, and $E\left[\left|b_{(k)}(l)\right|^{2}\right]=\sigma_{b}^{2}$. Even though we assume that $L_{g} \geq L_{h}$ for convenience, it is straightforward to find the MMSE solution when $L_{g}<L_{h}$ from the result with the assumption $L_{g} \geq L_{h}$.

The MSE can be written as

$$
M S E=E\left[\left\|\mathbf{d}^{T}(l) \mathbf{w}-b_{(1)}(l)\right\|^{2}\right] .
$$

Given that the decision is correct, the optimal solution is given by

$$
\mathbf{w}_{\text {mmse }}=\left(E\left[\mathbf{d}^{*}(l) \mathbf{d}^{T}(l)\right]\right)^{-1} E\left[\mathbf{d}^{*}(l) b_{(1)}(l)\right] .
$$

First, we consider

$$
E\left[\mathbf{d}^{*}(l) b_{(1)}(l)\right]=\left[\begin{array}{c}
E\left[\mathbf{u}_{(1)}^{*}(l) b_{(1)}(l)\right] \\
-E\left[\mathbf{v}_{(1)}^{*}(l) b_{(1)}(l)\right]
\end{array}\right] .
$$

Define

$$
\overline{\mathbf{h}}_{j}=[h_{j, 0} h_{j, 1} \cdot s h_{j, L_{h}} \underbrace{0, \ldots, 0}_{\left(L_{g}-L_{h}\right) \text { times }}]^{T} .
$$

Using (32), it can be shown that $E\left[u_{j, p,(1)}^{*}(l) b_{(1)}(l)\right]=$ $h_{j,-p}^{*} \sigma_{b}^{2}, p=0,-1, \ldots,-L_{g}$. Then, we have

$$
E\left[\mathbf{u}_{(1)}^{*}(l) b_{(1)}(l)\right]=\sigma_{b}^{2} \overline{\mathbf{h}}^{*}
$$

where $\overline{\mathbf{h}}=\left[\begin{array}{llll}\overline{\mathbf{h}}_{1}^{T} & \overline{\mathbf{h}}_{2}^{T} & \cdots & \overline{\mathbf{h}}_{L}^{T}\end{array}\right]^{T}$. The cross correlation of $v_{p,(1)}(l)$ and $b_{(1)}(l)$ is zero for $p=1,2, \ldots, L_{h}$. Hence, from (35), we have

$$
E\left[\mathbf{d}^{*}(l) \quad b_{(1)}(l)\right]=\sigma_{b}^{2} \overline{\mathbf{h}}_{0}^{*}
$$

where $\overline{\mathbf{h}}_{0}=\left[\begin{array}{ll}\overline{\mathbf{h}}^{T} & \underbrace{0, \ldots, 0}_{L_{h} \text { times }}\end{array}\right]^{T}$. Next, we need to find the following correlation matrix:

$$
\begin{aligned}
& E\left[\mathbf{d}^{*}(l) \mathbf{d}^{T}(l)\right] \\
& \quad=\left[\begin{array}{cc}
E\left[\mathbf{u}_{(1)}^{*}(l) \mathbf{u}_{(1)}^{T}(l)\right] & -E\left[\mathbf{u}_{(1)}^{*}(l) \mathbf{v}_{(1)}^{T}(l)\right] \\
-E\left[\mathbf{v}_{(1)}^{*}(l) \mathbf{u}_{(1)}^{T}(l)\right] & E\left[\mathbf{v}_{(1)}^{*}(l) \mathbf{v}_{(1)}^{T}(l)\right]
\end{array}\right] .
\end{aligned}
$$

To find $E\left[\mathbf{u}_{(1)}^{*} \mathbf{u}_{(1)}^{T}(l)\right]$, it can be shown in (38), at the bottom of the page. Since the background noise is white, we readily show that $A_{2}=N_{0} \delta_{s, t}$ in (38). From Assumption A2, we can show that

$$
\begin{aligned}
C\left(q, q^{\prime}, m, m^{\prime}\right)= & E\left[c_{(1), N l+q}^{*} c_{(1), N l+q^{\prime}} c_{(1), N l+m} c_{(1), N l+m^{\prime}}^{*}\right] \\
= & \frac{1}{N^{2}} \delta_{q, q^{\prime}} \delta_{m, m^{\prime}}+\frac{1}{N^{2}} \delta_{q, m} \delta_{q^{\prime}, m^{\prime}} \\
& -\frac{1}{N^{2}} \delta_{q, q^{\prime}} \delta_{m, m^{\prime}} \delta_{q, m}
\end{aligned}
$$

Furthermore, define $\bar{c}_{(1), N l+m}=\sum_{k=2}^{K} c_{(k), N l+m}$. Then, it follows that:

$$
\begin{aligned}
\bar{C}\left(q, q^{\prime}, m, m^{\prime}\right) & =E\left[\bar{c}_{(1), N l+q}^{*} \bar{c}_{(1), N l+q^{\prime}} c_{(1), N l+m} c_{(1), N l+m^{\prime}}^{*}\right] \\
& =\frac{K-1}{N^{2}} \delta_{q, q^{\prime}} \delta_{m, m^{\prime}} .
\end{aligned}
$$

Using (39), (40), and Assumption A1, we can show that

$$
\begin{aligned}
A_{1}= & \frac{\sigma_{b}^{2}}{N^{2}} \delta_{s,-p} \delta_{t,-p^{\prime}}+\sigma_{b}^{2}\left(1-\delta_{s,-p}\right)\left(1-\delta_{t,-p^{\prime}}\right) \\
& \times\left(C\left(m-s-p, m^{\prime}-t-p^{\prime}, m, m^{\prime}\right)\right. \\
& \left.\quad+\bar{C}\left(m-s-p, m^{\prime}-t-p^{\prime}, m, m^{\prime}\right)\right) .
\end{aligned}
$$

Substituting (41) into the first term on the right hand side (RHS) in (38), we have

$$
\begin{aligned}
& \sum_{m=0}^{N-1} \sum_{m^{\prime}=0}^{N-1} \sum_{p=0}^{L_{h}} \sum_{p^{\prime}=0}^{L_{h}} h_{j, p}^{*} h_{j^{\prime}, p^{\prime}} \delta_{s,-p} \delta_{t,-p^{\prime}} \sigma_{b}^{2} \\
& \quad+\sum_{m=0}^{N-1} \sum_{m^{\prime}=0}^{N-1} \sum_{p=0}^{L_{h}} \sum_{p^{\prime}=0}^{L_{h}} h_{j, p}^{*} h_{j^{\prime}, p^{\prime}}\left(1-\delta_{s,-p}\right)\left(1-\delta_{t,-p^{\prime}}\right) \\
& \quad \times C\left(m-s-p, m^{\prime}-t-p^{\prime}, m, m^{\prime}\right) \sigma_{b}^{2} \\
& =h_{j,-s}^{*} h_{j^{\prime},-t}+\frac{\sigma_{b}^{2}}{N} \\
& \quad \times\left(\sum_{p=0}^{L_{h}} h_{j, p}^{*} h_{j^{\prime}, p+(s-t)}-h_{j,-s}^{*} h_{j^{\prime},-t}\right) .
\end{aligned}
$$

For the second term on the RHS in (38), we similarly have

$$
\begin{aligned}
& \sum_{m=0}^{N-1} \sum_{m^{\prime}=0}^{N-1} \sum_{p=0}^{L_{h}} \sum_{p^{\prime}=0}^{L_{h}} h_{j, p^{*}} h_{j^{\prime}, p^{\prime}}\left(1-\delta_{s,-p}\right)\left(1-\delta_{t,-p^{\prime}}\right) \\
& \quad \times \bar{C}\left(m-s-p, m^{\prime}-t-p^{\prime}, m, m^{\prime}\right) \sigma_{b}^{2} \\
& =\frac{\sigma_{b}^{2}(K-1)}{N}\left(\sum_{p=0}^{L_{h}} h_{j, p}^{*} h_{j^{\prime}, p+(s-t)}-h_{j,-s}^{*} h_{j^{\prime},-t}\right) .
\end{aligned}
$$

$$
\begin{aligned}
E\left[u_{j, s,(1)}^{*}(l) u_{j^{\prime}, t,(1)}(l)\right]= & \sum_{m=0}^{N-1} \sum_{m^{\prime}=0}^{N-1} \sum_{p=0}^{L_{h}} \sum_{p^{\prime}=0}^{L_{h}} h_{j, p}^{*} h_{j^{\prime}, p^{\prime}} \underbrace{E\left[x_{N l+m-s-p}^{*} x_{N l+m^{\prime}-t-p^{\prime}} c_{(1), N l+m} c_{(1), N l+m^{\prime}}^{*}\right]}_{\triangleq_{A_{1}}} \\
& +\underbrace{\sum_{m=0}^{N-1} \sum_{m^{\prime}=0}^{N-1} E\left[n_{N l+m-s}^{*} n_{N l+m^{\prime}-t} c_{(1), N l+m} c_{(1), N l+m^{\prime}}^{*}\right]}_{\triangleq_{A_{2}}}, \\
s, t= & 0,-1, \ldots,-L_{g} .
\end{aligned}
$$


From (42) and (43), (38) is rewritten as

$$
\begin{gathered}
E\left[u_{j, s,(1)}^{*}(l) \quad u_{j^{\prime}, t,(1)}(l)\right]=\sigma_{b}^{2}\left(\frac{K}{N} \sum_{p=0}^{L_{h}} h_{j, p}^{*} h_{j^{\prime}, p+(s-t)}\right. \\
\left.+\frac{N-K}{N} h_{j,-s}^{*} h_{j^{\prime},-t}\right)+N_{0} \delta_{s, t} .
\end{gathered}
$$

Define the $\left(L_{g}+L_{h}+1\right) \times\left(L_{g}+1\right)$ Toeplitz matrix

$$
\mathcal{H}_{j}=\left[\begin{array}{cccc}
h_{j, 0} & & & \\
h_{j, 1} & h_{j, 0} & & \\
& & \ddots & \\
& \ddots & & \\
& & & h_{j, L_{h}}
\end{array}\right]
$$

and define

$$
\mathbf{R} \mathcal{H}=\left[\begin{array}{cccc}
\mathcal{H}_{1}^{T} \mathcal{H}_{1}^{*} & \mathcal{H}_{2}^{T} \mathcal{H}_{1}^{*} & \ldots & \mathcal{H}_{L}^{T} \mathcal{H}_{1}^{*} \\
\vdots & & & \\
& & \ddots & \\
\mathcal{H}_{1}^{T} \mathcal{H}_{L}^{*} & \mathcal{H}_{2}^{T} \mathcal{H}_{L}^{*} & \ldots & \mathcal{H}_{L}^{T} \mathcal{H}_{L}^{*}
\end{array}\right]
$$

so that

$$
E\left[\mathbf{u}_{(1)}^{*} \quad \mathbf{u}_{(1)}^{T}(l)\right]=\sigma_{b}^{2}\left(\frac{K}{N} \mathbf{R} \mathcal{H}+\frac{N-K}{N} \overline{\mathbf{h}}^{*} \overline{\mathbf{h}}^{T}\right)+N_{0} \mathbf{I} .
$$

We can find $E\left[\mathbf{u}_{(1)}^{*}(l) \quad \mathbf{v}_{(1)}^{T}(l)\right]$ using a similar approach as the one used above. After some manipulations, we have

$$
E\left[\mathbf{u}_{(1)}^{*}(l) \quad \mathbf{v}_{(1)}^{T}(l)\right]=\sigma_{b}^{2} \frac{K}{N} \mathcal{Z}^{*}
$$

where

$$
\mathcal{Z}=\left[\begin{array}{c}
\mathcal{Z}_{1} \\
\mathcal{Z}_{2} \\
\vdots \\
\mathcal{Z}_{L}
\end{array}\right] \text { and } \mathcal{Z}_{j}=\left[\begin{array}{cccc}
h_{j, 1} & h_{j, 2} & \cdots & h_{j, L_{h}} \\
h_{j, 2} & h_{j, 3} & & \\
\vdots & & & \\
0 & & &
\end{array}\right]
$$

In addition, it can be shown that

$$
E\left[\mathbf{v}_{(1)}^{*}(l) \quad \mathbf{v}_{(1)}^{T}(l)\right]=\sigma_{b}^{2} \frac{K}{N} \mathbf{I}
$$

From (46)-(48), we can find (37) in terms of the channel coefficients and noise variance. Then, together with (36), the optimal MMSE solution can be found to be

$$
\begin{aligned}
& \mathbf{w}_{\text {mmse }} \\
& =\left(\left[\begin{array}{cc}
\left(\frac{K}{N} \mathbf{R} \mathcal{H}+\frac{N-K}{N} \overline{\mathbf{h}}^{*} \overline{\mathbf{h}}^{T}+\frac{N_{0}}{\sigma_{b}^{2}} \mathbf{I}\right) & \frac{K}{N} \mathcal{Z}^{*} \\
\frac{K}{N} \mathcal{Z}^{T} & \frac{K}{N} \mathbf{I}
\end{array}\right]\right)^{-1} \overline{\mathbf{h}}_{0}^{*} .
\end{aligned}
$$

From (33), (34), and (36), the MMSE is given by

$$
\operatorname{MMSE}_{d f e}=\sigma_{b}^{2}\left(1-\mathbf{w}_{\mathrm{mmse}}^{H} \overline{\mathbf{h}}_{0}^{*}\right)
$$

In the above, we consider the case that the input of the FBF consists of all $K$ active spread signals. Now, let us assume that the sum of $K_{s}<K$ active spread signals including the pilot spread signal becomes the input signal for the FBF. In this case, it is shown that the following auto correlation and cross correlation matrices are modified to become

$$
\begin{aligned}
& E\left[\mathbf{u}_{(1)}^{*}(l) \quad \mathbf{v}_{(1)}^{T}(l)\right]=\sigma_{b}^{2} \frac{K_{s}}{N} \mathcal{Z}^{*} \\
& \text { and } E\left[\mathbf{v}_{(1)}^{*}(l) \quad \mathbf{v}_{(1)}^{T}(l)\right]=\sigma_{b}^{2} \frac{K_{s}}{N} \mathbf{I} \text {. }
\end{aligned}
$$

Hence, the optimal weight vector in (49) should be modified by using (51).

\section{ACKNOWLEDGMENT}

The authors are indebted to Associate Editor Dr. G. Leus and the reviewers for their constructive reviews for improving the quality of the paper.

\section{REFERENCES}

[1] M. Abdulrahman, A. U. H. Sheikh, and D. D. Falconer, "Decision feedback equalizer for CDMA in indoor wireless communications," IEEE J. Select. Areas Commun., vol. 12, pp. 698-706, May 1994.

[2] J. Choi, S. R. Kim, Y.-H. Jung, and Y. H. Lee, "Adaptive LMMSE receivers for CDMA systems over time-varying multipath fading channels," in Proc. 2001 IEEE Int. Conf. Communications, 2001, pp. 1955-1958.

[3] S. Chowdhury and M. D. Zoltowski, "Combined MMSE equalization and multi-user detection for high-speed CDMA forward link with sparse multipath channels," in Proc. 35th Asilomar Conf., 2001, pp. 389-393.

[4] C. D. Frank and E. Visotsky, "Adaptive interference suppression for direct-sequence CDMA systems with long spreading codes," presented at the 36th Allerton Conf., 1998.

[5] J. Hagenauer, E. Offer, and L. Papke, "Iterative decoding of binary block and convolutional codes," IEEE Trans. Inform. Theory, vol. 42, pp. 429-445, Mar. 1996.

[6] S. Haykin, Adaptive Filter Theory, 2/e. Englewood Cliffs, NJ: Prentice-Hall, 1991.

[7] K. Higuchi, A. Fujiwara, and M. Sawahashi, "Multipath interference canceller for high-speed packet transmission with adaptive modulation and coding scheme in W-CDMA forward link," IEEE J. Select. Areas Commun., vol. 20, pp. 419-432, Feb. 2002.

[8] M. Honig and M. K. Tsatsanis, "Adaptive techniques for multiuser CDMA receivers," IEEE Signal Processing Mag., vol. 17, pp. 49-61, May 2000.

[9] M. Honig, U. Madhow, and S. Verdu, "Blind adaptive multiuser detection," IEEE Trans. Inform. Theory, vol. 41, pp. 944-960, July 1995.

[10] A. Klein, "Data detection algorithms specially designed for the downlink of CDMA mobile radio systems," in Proc. IEEE VTC - 1997, 1997, pp. 203-207.

[11] T. P. Krauss, M. D. Zoltowski, and G. Leus, "Simple MMSE equalizers for CDMA downlink to restore chip sequence: Comparison to zeroforcing and rake," in Proc. IEEE ICASSP 2000, 2000, pp. 2865-2868.

[12] M. Latva-aho and M. Juntti, "LMMSE detection for DS-CDMA systems in fading channels," IEEE Trans. Commun., vol. 48, pp. 194-199, Feb. 2000.

[13] M. F. Madkour, S. C. Gupta, and Y.-P. E. Wang, "Successive interference cancellation algorithms for downlink W-CDMA communications," IEEE Trans. Wireless Commun., vol. 1, pp. 169-177, Jan. 2002.

[14] F. Petre, M. Moonen, M. Engels, B. Gyselinckx, and H. D. Man, "Pilotaided adaptive chip equalizer receiver for interference suppression in DS-CDMA forward link," in Proc. IEEE VTC-Fall 2000, 2000, pp. 303-308.

[15] P. B. Rapajic and B. S. Vucetic, "Adaptive receiver structures for asynchronous CDMA systems," IEEE J. Select. Areas Commun., vol. 12, pp. 685-697, May 1994.

[16] J. E. Smee and S. C. Schwartz, "Adaptive feedforward/feedback architectures for multiuser detection in high data rate wireless CDMA networks," IEEE Trans. Commun., vol. 48, pp. 996-1011, June 2000.

[17] M. K. Varanasi and B. Aazhang, "Multistage detection in asynchronous code-division multiple-access communications," IEEE Trans. Commun., vol. 38, pp. 509-519, Apr. 1990.

[18] S. Verdu, Multiuser Detection. Cambridge, U.K.: Cambridge Univ. Press, 1998. 
[19] X. Wang and H. V. Poor, "Blind equalization and multiuser detection in dispersive CDMA channels," IEEE Trans. Commun., vol. 46, pp. 91-103, Jan. 1998.

[20] Z. Xu and M. K. Tsatsanis, "Blind adaptive algorithms for minimum variance CDMA receivers," IEEE Trans. Commun., vol. 49, pp. 180-194, Jan. 2001.

[21] J. Yang and Y. Li, "A decision-feedback equalizer with tentative chip feedback for the downlink of wideband CDMA," in Proc. IEEE ICC 2002, 2002, pp. 119-123.

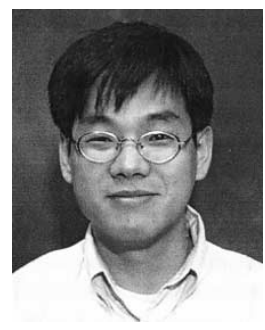

Jinho Choi (S'89-M'91-SM'02) was born in Seoul, Korea. He received the B.E. degree (magna cum laude) in electronics engineering from Sogang University, Seoul, in 1989, and the M.S.E. and Ph.D. degrees in electrical engineering from Korea Advanced Institute of Science and Technology (KAIST), Daejeon, in 1991 and 1994, respectively.

$\mathrm{He}$ is now with the School of Electrical Engineering and Telecommunications, The University of New South Wales, Sydney, Austrialia, as a Senior Lecturer. His research interests include wireless communications and array/statistical signal processing.

Dr. Choi was the recipient of the 1999 Best Paper Award of Signal Processing from EURASIP.

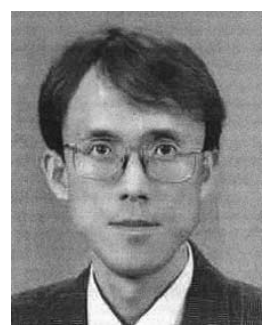

Seong Rag Kim (S'93-M'93) received the B.S. degree in electronics from the Kyungpook National University, Taegu, Korea, in 1981, and the M.S. degree in electrical engineering from the Korea Advanced Institute Science and Technology (KAIST), Seoul, in 1985, and the Ph.D. degree in electrical engineering and computer science from the University of Illinois, Chicago, in 1994.

In 1985, he joined the Electronics and Telecommunications Research Institute (ETRD), Taejon, Korea, where he is currently a Principal Member of Research Staff and the Project Manager of the multiuser detection for the W-CDMA. His current research interests are mainly concentrated on multiuser detection for DS-CDMA systems, smart antenna, MIMO, and non-Gaussian signal processing.

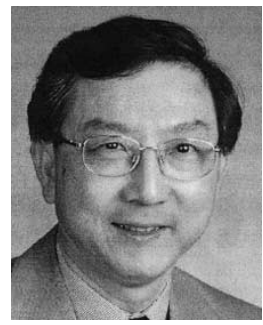

Cheng-Chew Lim (M'82-SM'02) received the B.Sc. and Ph.D. degrees from Loughborough University, Leicestershire, U.K.

His research interests include multichannel digital receivers, VLSI architectures for array processors, and control systems. He is with the School of Electrical and Electronic Engineering, University of Adelaide, Adelaide, Australia. 